\title{
Stability and Delay of Finite-User Slotted ALOHA With Multipacket Reception
}

\author{
Vidyut Naware, Student Member, IEEE, Gökhan Mergen, Student Member, IEEE, and Lang Tong, Fellow, IEEE
}

\begin{abstract}
The effect of multipacket reception (MPR) on stability and delay of slotted ALOHA based random-access systems is considered. A general asymmetric MPR model is introduced and the medium-access control (MAC) capacity region is specified. An explicit characterization of the ALOHA stability region for the two-user system is given. It is shown that the stability region undergoes a phase transition from a concave region to a convex polyhedral region as the MPR capability improves. It is also shown that after this phase transition, slotted ALOHA is optimal i.e., the ALOHA stability region coincides with the MAC capacity region. Further, it is observed that there is no need for transmission control when ALOHA is optimal i.e., ALOHA with transmission probability one is optimal. Next, these results are extended to a symmetric $N>2$ user ALOHA system. Finally, a complete characterization of average delay in capture channels for the two-user system is given. It is shown that in certain capture scenarios, ALOHA with transmission probability one is delay optimal for all stable arrival rates. Further, it is also shown that ALOHA with transmission probability one is optimal for stability and delay simultaneously in the two-user capture channel.
\end{abstract}

Index Terms-Capacity, delay, multipacket reception, random access, scheduling, slotted ALOHA, stability, wireless networks.

\section{INTRODUCTION}

\section{A. Motivation}

I T has been more than three decades since Abramson's landmark work on ALOHA [1]. Much of what we know about slotted ALOHA is based on the so-called collision model: a transmission is successful if and only if a single user transmits. While a deterministic collision model is accurate for wire-line communications, it is inadequate to model probabilistic receptions in wireless multiple access. Furthermore, advances in multiuser detection and space-time processing make it necessary to have a multipacket reception model that captures the ability of the receiver to decode simultaneous transmissions and the probabilistic nature of reception.

Manuscript received November 21, 2003; revised January 8, 2005. This work was supported in part by the Multidisciplinary University Research Initiative (MURI) under the Office of Naval Research Contract N00014-00-1-0564, the ARL CTA on Communications and Networks under Grant DAAD19-01-2-011, the National Science Foundation under Contract CCR-0311055, and the Army Research Office (ARO) under Grant ARO-DAAB19-00-1-0507. The material in this paper was presented in part at the Conference on Information Sciences and Systems, Baltimore, MD, March, 2003, the International Conference on Communications, Anchorage, AK, May, 2003, and the 41st Annual Allerton Conference, Monticello, IL, Oct., 2003.

V. Naware and L. Tong are with the School of Electrical and Computer Engineering at Cornell University, Ithaca, NY 14850 USA (e-mail: vidyut@ece. cornell.edu; ltong@ece.cornell.edu).

G. Mergen is with Qualcomm Incorporated, Campbell, CA 95008 USA (e-mail: gmergen@qualcomm.com).

Communicated by G. Sasaki, Associate Editor for Communication Networks. Digital Object Identifier 10.1109/TIT.2005.850060

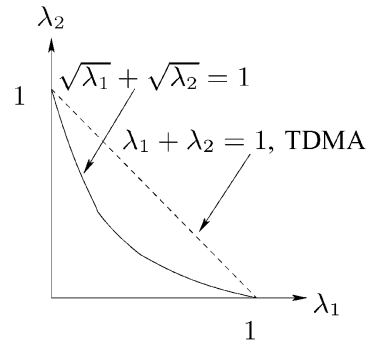

(a)

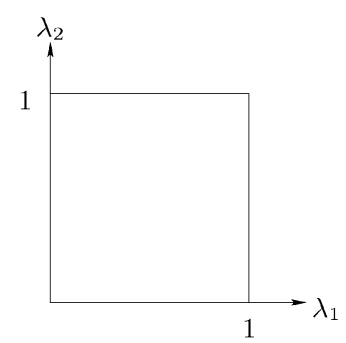

(b)
Fig. 1. Two-user stability region of slotted ALOHA for the collision channel and orthogonal channels. Solid lines represent the boundary of the ALOHA stability region. (a) Collision channel. (b) Orthogonal channel.

Insights into the effect of MPR on ALOHA can be gained by examining two extreme cases: the collision channel and the orthogonal channel. Fig. 1 shows the ALOHA stability regions of the two-user system for these cases. By stability region, we mean the set of arrival rates such that there exist transmission probabilities $\left(p_{1}, p_{2}\right) \in[0,1]^{2}$ under which the system is stable, in a sense to be made precise later. For the collision model, the stability region is not convex; an increase in the maximum rate of one user implies a disproportionate decrease of the other. As a random-access protocol, ALOHA is inferior to centralized time-division multiple access (TDMA) since its stability region is contained inside that of TDMA. To stabilize any point in the rate region, transmission control is necessary by choosing transmission probabilities carefully. The onus of handling multiuser interference rests entirely with the random-access protocol. The orthogonal channel, in contrast, models a physical layer that nullifies multiuser interference. As a result, the stability region takes the simple form of a unit square. There is no need for transmission control, and the rate for one user is independent of that of the other; ALOHA is optimal.

The orthogonal channel, of course, is not interesting for random access. What would be interesting are those cases when the multiuser interference affects the reception but not as severely as in the collision model. Can a distributed random access protocol such as ALOHA still be optimal? Is transmission control necessary? Is the stability region convex? A positive answer to the last question implies that given two stable rate pairs, all rate pairs on the line joining them are stable as well. What can we say about the performance of ALOHA for the general $N$-user system?

\section{B. Summary of Contributions}

We consider a general multipacket reception model. For each scheduled transmission, this model specifies a probability measure on the event space. We first give a complete 
characterization of the medium-access control (MAC) capacity region. By MAC capacity we mean the maximum throughput achievable by any MAC protocol without considering queue stability. We show that this region is a convex hull of a set of marginal probabilities. In particular, the MAC capacity region is specified only by the marginal probabilities of success of individual users.

We consider next the ALOHA stability region. Obviously, the ALOHA stability region is contained in the MAC capacity region. As already shown in Fig. 1, the ALOHA stability region is, in general, strictly smaller than the capacity region. We give a complete characterization for the two-user ALOHA system. We show that the stability region undergoes a distinct phase transition, from a nonconvex region to a convex polyhedral region, from a strict subset of the capacity region to the exact capacity region (thus, ALOHA is optimal). Furthermore, there is no need for transmission control once ALOHA is optimal. The same results hold for the symmetrical $N$-user system which has indistinguishable users with equal arrival rates. An inner bound for the general asymmetric $N$-user system is provided.

For a given rate vector, there are usually many transmission probabilities that stabilize the system. It is thus interesting to find the transmission probability that minimizes the average delay. We provide a complete delay characterization for the capture model in a symmetrical two-user system. Any nonzero probability of capture leads to a set of rates for which no transmission control minimizes the delay. As the probability of capture increases, the region of rates for which no transmission control minimizes the delay increases. As soon as the stability region becomes convex, no transmission control is delay optimal for all stable arrival rates.

\section{Related Work}

In spite of being such a simple random-access protocol, queueing theoretic analysis of ALOHA turns out to be extremely difficult under the collision model. Tsybakov and Mikhailov [2] initiated the stability analysis of finite-user slotted ALOHA. They found sufficient conditions for stability of the queues in the system using the principle of stochastic dominance. They found the stability region for the two-user case explicitly. For the symmetric case (viz. equal arrival rates for all terminals), they gave the maximum stable throughput. Rao and Ephremides [3] explicitly used the principle of stochastic dominance to find inner bounds to the stability region for the $N>2$ case. Szpankowski [4] found necessary and sufficient conditions for the stability of queues for a fixed transmission probability vector for the $N>2$ case. However, there is no simple computational procedure to verify these conditions since it involves the stationary joint queue statistics, which have not been found in closed form yet. Later, Luo and Ephremides [5] introduced the concept of instability ranks in queues to obtain tight inner and outer bounds on the stability region for the $N>2$ case. Interestingly, Anantharam [6] found the exact stability region of ALOHA for the finite-user case, albeit with a specific correlated arrival process. All the above stability results were derived for the collision channel only. And to date, there is no closed-form characterization of the stability region for the $N>2$ case (even for the collision channel with independent and identically distributed (i.i.d.) arrivals). The primary difficulty in analyzing this problem is the complex interactions among the queues.

The first attempt at analyzing ALOHA under multipacket reception was made by Ghez, Verdú, and Schwartz in [7], [8] under the infinite-user single-buffer model. Their multipacket reception (MPR) model was symmetrical in which users were indistinguishable. A special case of the symmetrical MPR model, but for finite users, was analyzed by Sant and Sharma [9]. They found a sufficient condition for stability with no transmission control. Adireddy and Tong [10] considered the effect of having knowledge of fading at the transmitters on the design of ALOHA. They showed that significant gains can be made by allowing the transmission probability to be a function of the channel state (as opposed to conventional power control). However, the MPR model that they used was symmetric with respect to the users while ours is not. A study of stability and capacity of general wireless networks for MPR models was presented in [11] where the MAC stability and capacity regions were characterized. In [11], the main focus was stability and capacity considerations with optimal MAC layer protocols whereas in this work we analyze performance of a specific random-access protocol viz., ALOHA. Protocols that exploit MPR have been proposed [10], [12], [13].

The remainder of this paper is organized as follows. In Section II, we specify the system model. In Section III, we define the notion of capacity region and in Section IV we define stability region. In Section V, we derive the stability region for the two-user case. We also characterize some interesting properties of this region. In Section VI, we provide stability results for the symmetric MPR case with $N>2$. We also give sufficient conditions for stability for the asymmetric MPR case with $N>2$. In Section VII, we apply our analytical results to three different receiver structures, viz., decorrelating, matched filter, and minimum mean-squared error (MMSE) and compare their performance in terms of the stability region to gain some insights. In Section VIII, we find expressions for delay and the optimizing transmission probability for the $N=2$ case for a subclass of MPR reception models. Finally, we conclude in Section IX.

\section{SYSTEM ModeL}

The system consists of $N$ users communicating with a common receiver. Each user has an infinite buffer for storing arriving and backlogged packets. The channel is slotted in time and a slot duration equals the packet transmission time. Packets are assumed to be of equal length for all the users. The arrivals at the $i$ th queue $(i \in\{1,2, \ldots, N\} \triangleq \mathcal{M})$ are i.i.d. Bernoulli random variables from slot to slot with mean $\lambda_{i}$. Arrival processes are assumed to be independent from user to user. If the $i$ th users' buffer is nonempty, he transmits a packet with probability $p_{i}$ in a slot.

A multiuser physical layer is assumed that allows the receiver to receive multiple packets simultaneously. Specifically, suppose that the set $\mathcal{S} \subseteq \mathcal{M}$ of users transmit in a slot, then we define for $\mathcal{R} \subseteq \mathcal{S}$, the conditional probability of reception by

$q_{\mathcal{R}, \mathcal{S}}=\operatorname{Pr}\{$ only packets from $\mathcal{R}$ are successfully received $\mid \mathcal{S}$ transmits $\}$. 
We assume that packet receptions are independent from slot to slot. Note that our reception model completely defines the probability space of packet receptions. The MPR reception model defined in [7] is symmetric with respect to the users and a special case of the above model. It follows that the marginal probability of success of $\mathcal{R}$ given that set $\mathcal{S}$ of users transmit is given by

$$
q_{\mathcal{R} \mid \mathcal{S}}=\sum_{\mathcal{U}: \mathcal{R} \subseteq \mathcal{U} \subseteq \mathcal{S}} q_{\mathcal{U}, \mathcal{S}}
$$

For example, consider the two-user case $N=2$. Then for $i=1,2$

$$
\begin{aligned}
& q_{i,\{i\}}= \operatorname{Pr}\{\text { user } i \text { is successful } \mid \text { only } \\
&\text { user } i \text { transmits }\} \operatorname{Pr}\{\text { only } \text { user } i \text { is successful } \mid \text { both } \\
& q_{i,\{1,2\}}=\text { users transmit }\} \\
& q_{\{1,2\},\{1,2\}}= \operatorname{Pr}\{\text { both users are successful } \mid \text { both } \\
&\text { users transmit }\}
\end{aligned}
$$

and the marginal probabilities of success are $(i=1,2)$

$$
q_{i \mid\{i\}}=q_{i,\{i\}}, \quad q_{i \mid\{1,2\}}=q_{i,\{1,2\}}+q_{\{1,2\},\{1,2\}} .
$$

We assume that the receiver gives an instantaneous feedback of all the packets that were successful in a slot at the end of the slot to all the users. The users remove successful packets from their buffers while unsuccessful packets are retained. It should be clear that the probabilities $q_{\mathcal{R}, \mathcal{S}}$ are a function of the receiver front end which will be employed by the receiver to "separate" users' signals.

Let $Q_{j}^{t}$ represent the queue length at the $j$ th buffer at the beginning of time slot $t$. Under the above system model, the $N$-dimensional process $Q^{t}=\left(Q_{1}^{t}, Q_{2}^{t}, \ldots, Q_{N}^{t}\right)$ is a Markov chain. The transition probability matrix of the Markov chain $\boldsymbol{Q}^{t}$ can be computed using the reception probabilities given by (1). Under mild conditions (for instance, $q_{i \mid i}>0$ for all $i \in \mathcal{M}), \boldsymbol{Q}^{t}$ is irreducible and aperiodic. We will assume $\boldsymbol{Q}^{t}$ to be an irreducible and aperiodic Markov chain throughout this paper. Let $\beta_{j}^{t}$ be the number of arrivals during the $t$ th slot to the $j$ th user with $\mathbb{E}\left(\beta_{j}^{t}\right)=\lambda_{j}$. Let the Bernoulli random variable $Y_{j}^{t}$ denote departures ${ }^{1}$ from queue $j$ in time slot $t$. Note that $Y_{j}^{t}$ captures the MPR receptions as well as the ALOHA random-access transmission of user $j$ in time slot $t$. Then the queue evolution for the $j$ th queue has the well known form [4]

$$
Q_{j}^{t+1}=\left[Q_{j}^{t}-Y_{j}^{t}\right]^{+}+\beta_{j}^{t}
$$

where $[x]^{+}$denotes $\max \{0, x\}$.

\section{MAC CAPACITY}

For the reception model defined by (1), we now define the notion of capacity region $(\mathfrak{C})$ of the network. Suppose that at $t=0$, all users in the network have infinitely many packets to send to the receiver. One may ask what possible long-term rates the reception model specified by (1) can support or achieve with

\footnotetext{
${ }^{1}$ The process $\left\{Y_{j}^{t}\right\}_{t=1}^{\infty}$ represents departures in the sense that $Y_{j}^{t}=1$ implies that a packet from queue $j$ was successfully received in slot $t$ only when $Q_{j}^{t}>0$. However, we could have $Y_{j}^{t}=1$ even when $Q_{j}^{t}=0$ [14].
}

optimal centralized scheduling. Here, we neglect the effects of source burstiness and thus the long-term "achievable" rates depend only on the reception model.

Let $\mathcal{R}_{S}(t)$ be the set of successful transmissions when the set of users $\mathcal{S}_{S}(t)$ transmit in slot $t$ under scheduling policy $S$. We allow the scheduling policy to be a function of the history of the network, viz., all the past arrivals and the packet success outcomes. The scheduling policy can be randomized as well.

Definition 1: A rate $\boldsymbol{\lambda}=\left(\lambda_{1}, \ldots, \lambda_{N}\right)$ is called achievable if there exists a scheduling policy $\left(S^{*}\right)$ with delivery rate at least $\lambda$, i.e.,

$$
\liminf _{T \rightarrow \infty} \frac{1}{T} \sum_{t=0}^{T-1} \mathbf{1}\left(i \in \mathcal{R}_{S^{*}}(t)\right) \geq \lambda_{i} \quad \forall i \quad \text { a.s. }
$$

Capacity region $(\mathfrak{C})$ is the closure of the set of all achievable rates.

This notion of "achievable" rate has been used before in [11], [15]. The following theorem provides a simple way to compute $\mathfrak{C}$ in terms of the marginal probabilities of success of each user.

Theorem 1: A rate $\lambda$ is achievable if and only if there exists a probability measure $\{p(\mathcal{S}): \mathcal{S} \subseteq \mathcal{M}\}$ such that $\forall i \in \mathcal{M}$

$$
\lambda_{i} \leq \sum_{\mathcal{S} \subseteq \mathcal{M}} q_{i \mid \mathcal{S}} p(\mathcal{S}) \text {. }
$$

Proof: Refer to [14].

The above result shows that $\mathfrak{C}$ is the convex hull of the $N$-tuples consisting of the marginal reception probabilities of the users in all possible $2^{N}$ transmission scenarios. Intuitively, the achievability part of the proof follows by observing that if a scheduler chooses the subset of transmitting users $\mathcal{S}$ with probability $p(\mathcal{S})$ i.i.d. in every slot, then $\boldsymbol{\lambda}$ satisfying (7) is achievable. Note that a direct consequence of the above theorem is that the capacity region is convex.

Fig. 2 shows the two-user capacity region for two different reception models. Clearly, the convex hull can take only two possible forms; either it is a triangle (Fig. 2(a)) or it is a quadrilateral (Fig. 2(b)). For the case of Fig. 2(a), optimal scheduling is equivalent to TDMA where to achieve any rate in the capacity region, it suffices to allow only one user to transmit in a slot. On the other hand in the case of Fig. 2(b), the scheduler has to consider allowing both users to transmit simultaneously to achieve some rate pairs.

\section{MAC STABILITY REGION}

Before we proceed to derive some of the results of the next section, a few definitions are in order. We use the definition of stability used by Szpankowski [4].

Definition 2: A multidimensional stochastic process $\boldsymbol{Q}^{t}=$ $\left(Q_{1}^{t}, \ldots, Q_{N}^{t}\right)$ is stable if for $\boldsymbol{x} \in \mathbb{N}^{N}$ the following holds:

$$
\lim _{t \rightarrow \infty} \operatorname{Pr}\left\{\boldsymbol{Q}^{t}<\boldsymbol{x}\right\}=F(\boldsymbol{x}) \text { and } \lim _{\boldsymbol{x} \rightarrow \infty} F(\boldsymbol{x})=1 .
$$

If a weaker condition holds, viz.,

$$
\lim _{\boldsymbol{x} \rightarrow \infty} \liminf _{t \rightarrow \infty} \operatorname{Pr}\left\{\boldsymbol{Q}^{t}<\boldsymbol{x}\right\}=1
$$




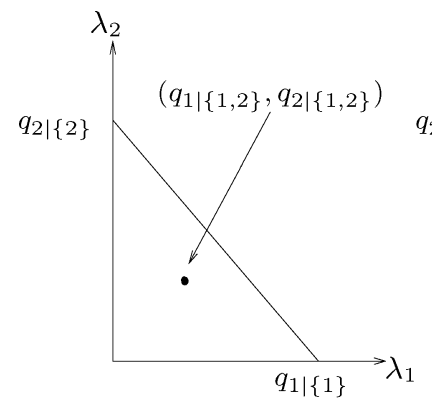

(a)

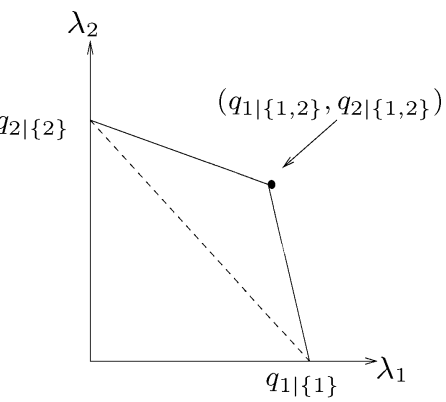

(b)
Fig. 2. $\quad \mathfrak{C}$ (two-user case) under different reception models. (a) $\frac{q_{1 \mid\{1,2\}}}{q_{1 \mid\{1\}}}+$ $\frac{q_{2 \mid\{1,2\}}}{q_{2 \mid\{2\}}}<1$. (b) $\frac{q_{1 \mid\{1,2\}}}{q_{1 \mid\{1\}}}+\frac{q_{2 \mid\{1,2\}}}{q_{2 \mid\{2\}}}>1$.

then the process is called substable. Further, the process is said to be unstable if it is not substable.

The related concepts of stability and substability have been well studied (see [4], [5]). For a queueing system, stability can be interpreted as the convergence of the queue lengths in distribution to a proper random variable (viz., a random variable that is finite with probability one) or, equivalently, the existence of a proper limiting distribution. As mentioned before, with ALOHA, the queue process $Q^{t}$ is an aperiodic and irreducible Markov chain on a countable state space. It can be shown that for $\boldsymbol{Q}^{t}$, the notions of stability and substability are equivalent and stability is equivalent to the existence of a unique stationary distribution (see [16]). Though the transition matrix of $\boldsymbol{Q}^{t}$ depends on the reception probabilities given by (1), we will see that the stability properties of $Q^{t}$ can be characterized with only the marginal probabilities of success of users $\left(\left\{q_{i \mid \mathcal{T}}: \mathcal{T} \subseteq \mathcal{M}, i=1, \ldots, N\right\}\right)$.

It would be natural to expect the stability of a queueing system to depend on the average arrival rate and average service rate. This intuition is made concrete by the Loynes' theorem [17] which says that if the arrival and departure processes of a queue are strictly stationary and ergodic then i) the queue is stable if the average arrival rate is less than the average departure rate and ii) the queue is unstable if the average arrival rate exceeds the average departure rate. This motivates the following characterization of stability.

Definition 3: For an $N$-user multiple-access system with a given MAC protocol and arrival process distribution, the stability region is defined as the closure ${ }^{2}$ of the set of arrival rates $\boldsymbol{\lambda}=\left[\lambda_{1}, \lambda_{2}, \ldots, \lambda_{N}\right]$ for which the queues in the system are stable.

In particular, for the $N$-user slotted ALOHA system defined in Section II, the stability region is defined as the set of arrival rates $\boldsymbol{\lambda}=\left[\lambda_{1}, \lambda_{2}, \ldots, \lambda_{N}\right]$ for which there exists a transmission probability vector $\boldsymbol{p}=\left[p_{1}, p_{2}, \ldots, p_{N}\right]$ such that the queues in the system are stable. We will denote the stability region of

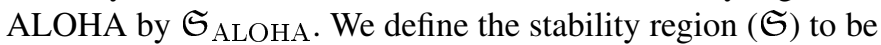

\footnotetext{
${ }^{2}$ Generally, it is difficult to characterize stability on the boundary of the stability region. The set operation of closure allows us to conveniently get around stability properties of points on the boundary of the stability region.
}

the union of the stability regions over all MAC protocols (for the reception model given by (1)).

The capacity region characterizes the set of departure rates that are supported by centralized scheduling whereas the stability region provides the set of stable arrival rates with all MAC protocols. Here, note that we also consider MAC protocols with memory, viz., the MAC scheme can allow users to transmit based on the past history of the channel outcomes. Intuitively, we expect the stability region of any MAC protocol to be contained within the capacity region since in a stable system, the arrival rate is equal to the departure rate ${ }^{3}$.

Theorem 2: For the $N$-user random-access system with reception model specified by (1), $\mathfrak{S} \subseteq \mathfrak{C}$.

\section{Proof: Refer to [14].}

Thus, $\mathfrak{C}$ provides a simple easily computable upper bound to $\mathfrak{S}_{\text {ALOHA }}$. However, unlike the capacity region, the stability region of ALOHA is not easy to characterize. We have the following relation: $\mathfrak{S}_{\mathrm{ALOHA}} \subseteq \mathfrak{S} \subseteq \mathfrak{C}$.

\section{Stability AND Optimality of ALOHA fOR $N=2$}

\section{A. Stability Region of ALOHA}

We first find the stability region for the $N=2$ case for the general reception model given by (3). We will show that the marginal probabilities given by (4) are alone sufficient to characterize the stability region of ALOHA.

Define $Q_{1} \triangleq q_{1 \mid\{1\}}-q_{1 \mid\{1,2\}}, Q_{2} \triangleq q_{2 \mid\{2\}}-q_{2 \mid\{1,2\}}$, and $\boldsymbol{q} \triangleq\left[q_{1 \mid\{1\}}, q_{2 \mid\{2\}}, q_{1 \mid\{1,2\}}, q_{2 \mid\{1,2\}}\right.$. Thus, $Q_{1}$ and $Q_{2}$ denote the difference between the (conditional) probability of success in the absence of interference and the (conditional) probability of success in the presence of interference for the users. For the collision channel, $Q_{1}=Q_{2}=1$ whereas for orthogonal channels, $Q_{1}=Q_{2}=0$.

To find the stability region, we first find the stability region of the ALOHA system for a fixed transmission probability vector $\boldsymbol{p}\left(=\left[p_{1}, p_{2}\right]\right)$. The following lemma gives us exactly that.

Lemma 1: If $Q_{1} \geq 0$ and $Q_{2} \geq 0$, the stability region of slotted ALOHA for the general packet reception model for a given $\left[p_{1}, p_{2}\right]\left(\mathfrak{S}_{\mathrm{ALOHA}}(\boldsymbol{p})\right)$ is given by

$$
\lambda_{1} \leq p_{1} q_{1 \mid\{1\}}-\frac{p_{1} p_{2} \lambda_{2} Q_{1}}{\lambda_{2}^{*}}, \quad \text { for } \lambda_{2} \leq \lambda_{2}^{*}
$$

and

$$
\lambda_{2} \leq p_{2} q_{2 \mid\{2\}}-\frac{p_{1} p_{2} \lambda_{1} Q_{2}}{\lambda_{1}^{*}}, \quad \text { for } \lambda_{1} \leq \lambda_{1}^{*}
$$

where

$$
\lambda_{1}^{*}=p_{1} q_{1 \mid\{1\}}-p_{1} p_{2} Q_{1} \text { and } \lambda_{2}^{*}=p_{2} q_{2 \mid\{2\}}-p_{1} p_{2} Q_{2} .
$$

Proof: We use the idea of stochastic dominance and an argument similar to that by Rao and Ephremides [3]. Refer to the Appendix for details.

\footnotetext{
${ }^{3}$ In fact, in [18], it is shown that under certain general conditions on the reception model, $\mathfrak{S}=\mathfrak{C}$. Further, there exist cases when $\mathfrak{S} \subset \mathfrak{C}$. A simple example can be found in [18].
} 


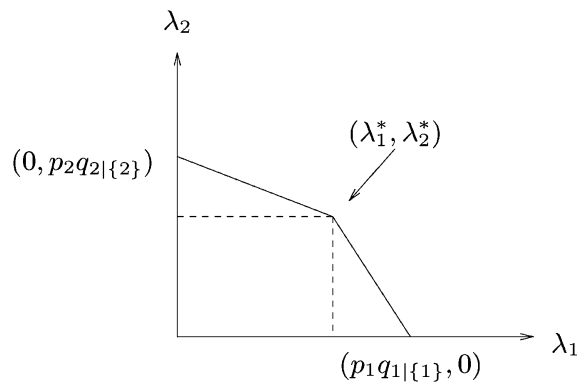

Fig. 3. Stability region for a fixed transmission probability vector $\left[p_{1}, p_{2}\right]$.

The stability region of ALOHA with fixed $\left[p_{1}, p_{2}\right]$ for the following well-known cases are as follows.

a) Orthogonal channel: In this case, $Q_{1}=Q_{2}=0$. By Lemma 1, the stability region is the two-dimensional box in the nonnegative quadrant bounded by the lines $\lambda_{1}=0$, $\lambda_{2}=0, \lambda_{1}=p_{1} q_{1 \mid\{1\}}$. and $\lambda_{2}=p_{2} q_{2 \mid\{2\}}$.

b) Collision channel: Since $Q_{1}=Q_{2}=1$ in this case, $\lambda_{1}^{*}=p_{1}\left(1-p_{2}\right)$ and $\lambda_{2}^{*}=p_{2}\left(1-p_{1}\right)$. The stability region is

$$
\lambda_{1} \leq p_{1}\left(1-\frac{\lambda_{2}}{\left(1-p_{1}\right)}\right), \quad \text { for } \lambda_{2} \leq p_{2}\left(1-p_{1}\right)
$$

and

$$
\lambda_{2} \leq p_{2}\left(1-\frac{\lambda_{1}}{\left(1-p_{2}\right)}\right), \quad \text { for } \lambda_{1} \leq p_{1}\left(1-p_{2}\right) .
$$

Irrespective of the reception model, the stability region for a given $\boldsymbol{p}$ has a form as shown in Fig. 3. The conditions $Q_{1} \geq$ 0 and $Q_{2} \geq 0$ are equivalent to the probability of success of any user in the presence of interference (from the other user) be no greater than the probability of success in the absence of interference-a reasonable and practical assumption. Note that although Fig. 3 shows the stability region for a fixed $\boldsymbol{p}$ to be convex, it need not be convex as $\boldsymbol{p}$ varies over $[0,1]^{2}$. It follows from Lemma 1 directly that the stability region of ALOHA in the two-user case depends only on the marginal probabilities of success of the users since $Q_{1}$ and $Q_{2}$ depend only on the marginals.

Using

$$
\mathfrak{S}_{\mathrm{ALOHA}}=\bigcup_{\boldsymbol{p} \in[0,1]^{2}} \mathfrak{S}_{\mathrm{ALOHA}}(\boldsymbol{p})
$$

we give a complete description of the stability region of ALOHA with the following lemma.

Lemma 2: If $Q_{1} \geq 0$ and $Q_{2} \geq 0$, then the stability region of slotted ALOHA ( $\left.\mathfrak{S}_{\text {ALOHA }}\right)$ for the general reception model is given by $\mathcal{R}_{1} \cap \mathcal{R}_{2}$ where

$$
\begin{aligned}
\mathcal{R}_{1} \triangleq\{ & \left(\lambda_{1}, \lambda_{2}\right):\left(\lambda_{1}, \lambda_{2}\right) \geq(0,0),\left(\lambda_{1}, \lambda_{2}\right) \text { lies } \\
& \text { below the curve } \left.\lambda_{2}=f\left(\lambda_{1} ; q_{1 \mid\{1\}}, q_{2 \mid\{2\}}, Q_{1}, Q_{2}\right)\right\}
\end{aligned}
$$

and

$$
\begin{aligned}
\mathcal{R}_{2} \triangleq & \left\{\left(\lambda_{1}, \lambda_{2}\right):\left(\lambda_{1}, \lambda_{2}\right) \geq(0,0),\left(\lambda_{1}, \lambda_{2}\right)\right. \text { lies } \\
& \text { below the curve } \left.\lambda_{1}=f\left(\lambda_{2} ; q_{2 \mid\{2\}}, q_{1 \mid\{1\}}, Q_{2}, Q_{1}\right)\right\}
\end{aligned}
$$

where

$$
f(\lambda ; \alpha, \beta, \gamma, \delta)= \begin{cases}\beta-\frac{\lambda \delta}{\alpha-\gamma}, & \lambda \in \mathcal{I}_{1} \\ \frac{(\sqrt{\alpha \beta}-\sqrt{\lambda \delta})^{2}}{\gamma}, & \lambda \in \mathcal{I}_{2}\end{cases}
$$

where

$$
\mathcal{I}_{1}=\left[0, \frac{\beta(\alpha-\gamma)^{2}}{\alpha \delta}\right] \text { and } \mathcal{I}_{2}=\left(\frac{\beta(\alpha-\gamma)^{2}}{\alpha \delta}, \frac{\alpha \beta}{\delta}\right] .
$$

If either $Q_{1}$ or $Q_{2}$ equals zero, then we assume $\frac{1}{0}=\infty$ and the result still holds.

Proof: Refer to the Appendix.

We note a few interesting properties about the stability region. First, the function $f$ characterizing the stability region in (16) is linear for some part of the domain $\mathcal{I}_{1}$ and is strictly convex in the remainder of its domain $\mathcal{I}_{2}$. The stability region for the two-user collision channel can be found as a special case with $q_{1 \mid\{1\}}=1, q_{2 \mid\{2\}}=1, q_{1 \mid\{1,2\}}=0$, and $q_{2 \mid\{1,2\}}=0$ and it is bounded by the curve $\sqrt{\lambda_{1}}+\sqrt{\lambda_{2}}=1$, which is strictly convex everywhere. In fact, it is easy to see that the interval where $f$ is linear has nonzero Lebesgue measure as soon as there is a nonzero probability of success in the presence of interference, i.e., $q_{1 \mid\{1\}}-Q_{1}=q_{1 \mid\{1,2\}}>0$. Thus, there is a characteristic change in the structure of the stability region as soon as we have multipacket reception. Second, we see that there is a symmetry in the way the two regions $\mathcal{R}_{1}$ and $\mathcal{R}_{2}$ are defined in terms of the function $f$.

We now provide one of the main results that gives a structural characterization of the ALOHA stability region.

Theorem 3: Let $Q_{1} \geq 0$ and $Q_{2} \geq 0$. Assume $q>0$, i.e., nonzero probability of success in the presence and absence of interference. Then, the following are equivalent.

1) $\mathfrak{S}_{\mathrm{ALOHA}}$ is convex.

2) $\mathfrak{S}_{\mathrm{ALOHA}}$ is a polyhedron.

3) The marginal reception probabilities satisfy

$$
\mathcal{D}(\boldsymbol{q}) \triangleq \frac{q_{1 \mid\{1,2\}}}{q_{1 \mid\{1\}}}+\frac{q_{2 \mid\{1,2\}}}{q_{2 \mid\{2\}}} \geq 1 .
$$

4) $\mathfrak{S}_{\mathrm{ALOHA}}=\mathfrak{S}=\mathfrak{C}$.

5) $\boldsymbol{p}=[1,1]$ is optimal in the sense that $\mathfrak{S}_{\mathrm{ALOHA}}=$ $\mathfrak{S}_{\text {ALOHA }}([1,1])$.

If $\mathfrak{S}_{\text {ALOHA }}$ is nonconvex, then it is bounded by lines close to the axes and by a strictly convex function in the interior.

Proof: Refer to the Appendix.

Fig. 4 shows the stability regions characterized by the $q$ vector as given by Theorem 3. For the collision channel, the stability region is nonconvex and bounded by a strictly convex curve. As soon as there is (weak) MPR, the stability region is bounded by lines near the axes and a nonlinear strictly convex function elsewhere. After a certain critical MPR level $(\mathcal{D}(\boldsymbol{q})=1)$ is reached, the stability region becomes a convex polyhedron. Thus, there is a critical point for the $q$ vector at which the behavior of the stability region makes a phase transition from a very complex form to a much more simpler form (a quadrilateral). Further, this critical point depends only on the sum of the ratios of probability of success of users in the presence of interference to that in the absence of interference. 


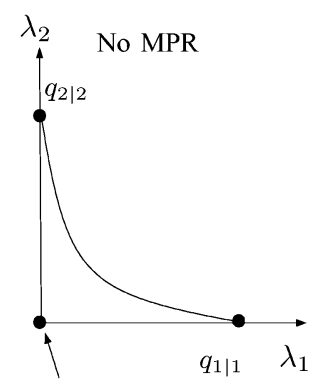

$\left(q_{1 \mid\{1,2\}}, q_{2 \mid\{1,2\}}\right)$

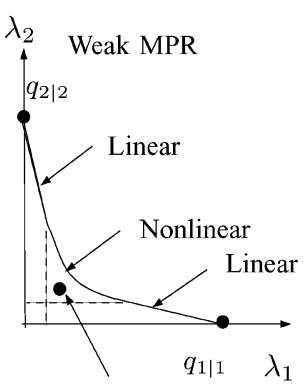

$\left(q_{1 \mid\{1,2\}}, q_{2 \mid\{1,2\}}\right)$

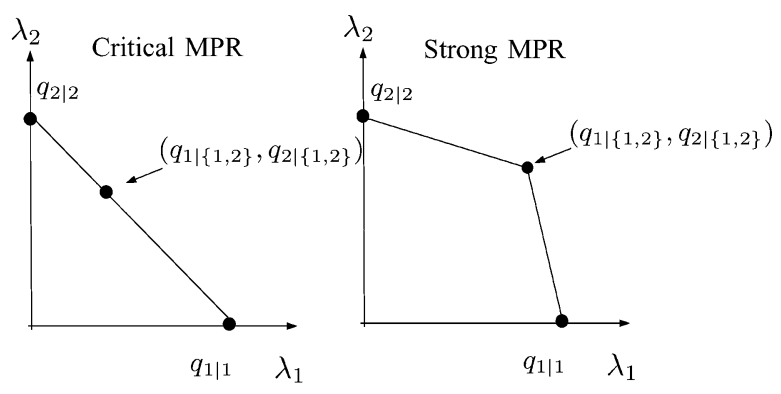

Fig. 4. $\mathfrak{S}_{\mathrm{ALOHA}}$ for different reception models with $q_{1 \mid\{1\}}$ and $q_{2 \mid\{2\}}$ fixed.

The condition of the stability region being a convex polyhedron corresponds to a regime in which when one user increases his rate, the other user's maximum supportable rate decreases linearly, and that too at a rate which is low until a certain point and then suddenly increases. Another interpretation is that when the stability region is convex then higher sum rates can be achieved. In addition, when the stability region is convex we know that if two rate pairs are stable then any rate pair lying on the line segment joining those two rate pairs is also stable. When equality holds in (18), the stability region is a triangle as shown in Fig. 4. All the rate pairs in this region can be stabilized by TDMA schemes (even in a collision channel). Thus, the condition $\frac{q_{1 \mid\{1,2\}}}{q_{1 \mid\{1\}}}+\frac{q_{2 \mid\{1,2\}}}{q_{2 \mid\{2\}}}>1$ gives us the regime in which a distributed strategy like slotted ALOHA can do better than a TDMA scheme.

When $\mathfrak{S}_{\text {ALOHA }}$ is not a polyhedron, it has a much more complex form. This is also the regime in which the stability region is not convex. In this regime, when one user increases his rate the other user's rate has to be reduced drastically in order to keep the system stable.

\section{B. Optimality of ALOHA}

Equivalence conditions three and four in Theorem 3 specify the regime of MPR capability where slotted ALOHA is optimal. $\mathfrak{S}_{\text {ALOHA }}=\mathfrak{S}$ implies that ALOHA can stabilize all rates that can be stabilized by any centralized or decentralized MAC protocol. Note that the point from where slotted ALOHA is optimal coincides with the phase transition point of the ALOHA stability region.

In order to stabilize a rate within the stability region of ALOHA, one has to choose an appropriate transmission probability $\boldsymbol{p}$ which, in general, is a function of the arrival rate. But the surprising observation when the stability region is convex, is that $\mathfrak{S}_{\mathrm{ALOHA}}=\mathfrak{S}_{\mathrm{ALOHA}}([1,1])$. This implies that when the stability region is convex, both users should always transmit packets (if they have any) to stabilize any stabilizable rate and no transmission control is required. We call this degenerate instance of ALOHA "persistent ALOHA." Note that with centralized scheduling, to stabilize a particular rate the scheduler has to allocate a proportion of time for each possible subset of transmitting users. But the preceding result implies that there is no need for "scheduling" any transmissions. The strategy-transmit if you have packets-will do. The reason for this is that the users' queues empty out ever so often as a

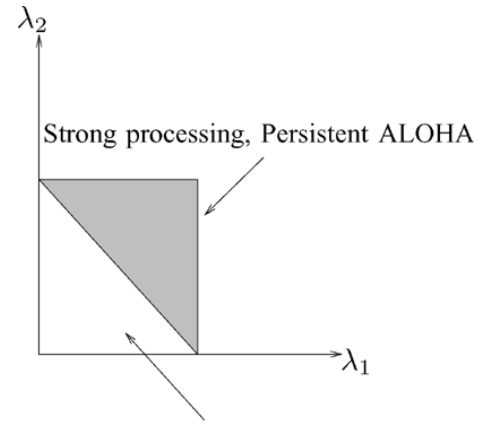

Weak processing, Need scheduling

Fig. 5. Regions showing optimal allocation of resources to PHY and MAC layer for all MPR models.

result of which there is a proportion of time when the users are transmitting alone. This pseudo scheduling of users automatically takes care of stabilizing the queues for the particular arrival rates.

The implication for cross-layer design is clear-if we can design a reasonably strong physical layer, then there is no need for a sophisticated MAC layer. Intuitively, it is quite clear that as the ability of the physical layer to orthogonalize users increases, then the need for random-access protocols does not arise. However, surprisingly we find that the point at which we could dispense the MAC layer comes well before we have an ideal physical layer. Equation (18) gives both the metric for measuring the MPR capability and the condition under which the MAC layer is dispensable.

Fig. 5 shows how the knowledge of MPR capability can help in designing a better MAC layer. In a low-MPR regime, the physical layer is weak; a larger amount of resources should be allocated to the MAC layer. On the other hand, if we allocate more resources to the physical layer (with advanced signal processing) thereby guaranteeing a strong MPR channel, no resources are needed at the MAC layer; persistent ALOHA is optimal.

\section{Stability of ALOHA FOR the $N>2$ CASE}

Little progress has been made in giving an exact characterization of the stability region of ALOHA for the $N>2$ case. In this section, for a symmetric MPR channel, we provide conditions under which persistent ALOHA is optimal among all MAC protocols. For the more general asymmetric MPR channel, we provide sufficient conditions for stability. 


\section{A. Symmetric MPR, Symmetric Arrivals Case}

For completeness, we first provide the symmetric MPR reception model introduced by Ghez, Verdú, and Schwartz [7]. Multipacket reception is parametrized by a matrix $C$ whose entries are given by

$C_{n, k}=\operatorname{Pr}\{k$ packets are successfully received $\mid n$ packets are transmitted $\}$.

Thus, we can write $\boldsymbol{C}$ as

$$
\boldsymbol{C}=\left(\begin{array}{cccc}
C_{1,0} & C_{1,1} & & \\
C_{2,0} & C_{2,1} & C_{2,2} & \\
\vdots & \vdots & \vdots & \ddots
\end{array}\right)
$$

This channel model is general enough to model the collision channel and the capture channel as special cases. The corresponding MPR matrices for the collision channel, and the capture channel are, respectively,

$$
\left(\begin{array}{cccc}
0 & 1 & 0 & \ldots \\
1 & 0 & 0 & \ldots \\
\vdots & \vdots & \vdots & \ddots
\end{array}\right), \quad\left(\begin{array}{cccc}
0 & 1 & 0 & \ldots \\
1-b_{2} & b_{2} & 0 & \ldots \\
1-b_{3} & b_{3} & 0 & \ldots \\
\vdots & \vdots & \vdots & \ddots
\end{array}\right)
$$

where $b_{i}$ denotes the probability of capture given $i$ simultaneous transmissions. We also define

$$
C_{n} \triangleq \sum_{k=1}^{n} k C_{n, k}
$$

which is the expected number of correctly received packets given $n$ packets are transmitted. By symmetry of this reception model, it follows that $C_{i} / i$ is the marginal probability of success of any one of the $i$ users that transmit in a slot.

Consider an $N$-user symmetric system with each user having an arrival rate $\frac{\lambda}{N}$. Let $Q^{t}$ be the $N$-tuple representing the queue lengths of users at time $t$. Given a reception model and a MAC protocol, we can define the maximum stable throughput to be the supremum of all arrival rates $\lambda$ such that $\boldsymbol{Q}^{t}$ is stable. For example, the maximum stable throughput of ALOHA for a given transmission probability $p$ (denoted by $\rho_{\mathrm{ALOHA}}(p)$ ) is the supremum of all arrival rates $\lambda$ such that $\boldsymbol{Q}^{t}$ is stable. Further, we define the maximum stable throughput of ALOHA to be

$$
\rho_{\mathrm{ALOHA}}=\sup _{p \in[0,1]} \rho_{\mathrm{ALOHA}}(p) .
$$

Let $\rho$ denote the supremum of the maximum stable throughput over all MAC protocols. By definition, $\rho_{\mathrm{ALOHA}} \leq \rho$. We also have the following.

Corollary 1 (To Theorem 2): For the $N$-user symmetric system with symmetric MPR reception model given by (19)

$$
\rho \leq \max \left\{C_{1}, C_{2}, \ldots, C_{N}\right\} .
$$

Proof: By Theorem 2, for any stable arrival rates $\left\{\lambda_{i}=\frac{\lambda}{N}: i \in \mathcal{M}\right\}$, there exists a probability measure $\{p(\mathcal{S}): \mathcal{S} \subseteq \mathcal{M}\}$ such that

$$
\lambda_{i}=\frac{\lambda}{N} \leq \sum_{\mathcal{S}} q_{i \mid \mathcal{S}} p(\mathcal{S}) \mathbf{1}[i \in \mathcal{S}] .
$$

Summing over $i$ gives

$$
\begin{aligned}
\lambda & \leq \sum_{i=1}^{N} \sum_{\mathcal{S}} q_{i \mid \mathcal{S}} p(\mathcal{S}) \mathbf{1}[i \in \mathcal{S}] \\
& =\sum_{i=1}^{N} \sum_{\mathcal{S}} \frac{C_{|\mathcal{S}|}}{|\mathcal{S}|} p(\mathcal{S}) \mathbf{1}[i \in \mathcal{S}] \\
& =\sum_{\mathcal{S}} \frac{C_{|\mathcal{S}|}}{|\mathcal{S}|} p(\mathcal{S}) \sum_{i=1}^{N} \mathbf{1}[i \in \mathcal{S}] \\
& =\sum_{\mathcal{S}} C_{|\mathcal{S}|} p(\mathcal{S}) \\
& \leq \max \left\{C_{1}, \ldots, C_{N}\right\}
\end{aligned}
$$

where (22) follows from user and MPR channel symmetry. Thus, the maximum stable throughput of any MAC protocol is upper-bounded by $\max \left\{C_{1}, \ldots, C_{N}\right\}$.

Since for some $i, C_{i}=\max \left\{C_{1}, \ldots, C_{N}\right\}$, we can conclude that a sum rate of $C_{i}$ can be achieved by centralized scheduling of all sets consisting of $i$ users. The above result is analogous to the result $\mathfrak{S} \subseteq \mathfrak{C}$ for the two user case.

The following theorem extends Theorem 3 to the symmetric $N>2$ case.

Theorem 4: For the symmetric MPR channel, let

$$
C_{1} \geq \frac{C_{2}}{2} \geq \cdots \geq \frac{C_{i}}{i} \geq \cdots \geq \frac{C_{N}}{N} .
$$

Then, the following are equivalent.

1) The reception probabilities satisfy

$$
C_{N}=\max \left\{C_{1}, \ldots, C_{N}\right\}
$$

2) $\rho_{\mathrm{ALOHA}}(1)=\rho_{\mathrm{ALOHA}}=\rho=\max \left\{C_{1}, \ldots, C_{N}\right\}$.

Proof: Refer to the Appendix.

Theorem 4 shows that there is a regime for which ALOHA with transmission probability one, i.e., persistent ALOHA is optimal among all MAC protocols. Equation (23) is equivalent to the condition that the probability of packet success per user decreases as interference increases. Equation (24) ensures that the expected number of successful receptions is maximized when all users transmit.

Note that for the orthogonal channel case, $C_{i}=i C_{1}$ and obviously persistent ALOHA is optimal. As in the two user case, we see that persistent ALOHA is optimal for a much larger class of symmetric MPR channels as specified by (24) and (23).

It is interesting to compare our results with those of [19] in which the problem of scheduling transmissions for the downlink of a multiple-antenna cellular system is considered. Viswanath, Tse, and Laroia show that from an information-theoretic point of view, a good strategy for the base station is to employ "dumb" antennas (in the sense of not doing any signal processing other than that in a single-antenna system) and implement "smart" scheduling (in the sense of scheduling users who have the best channel at that time). Thus, they show that more resources should be allocated to scheduling than to the physical layer for the downlink. Our problem is in some sense, 
a conceptual dual of the downlink problem. Our results apply to the uplink of a multiple-antenna cellular system, and since we wish to address source burstiness, we choose the framework of random access. In contrast to [19], our results show the tradeoff involved in allocation of resources to the MAC and the physical layer.

Apart from the symmetric case, we cannot say whether such a result would carry over to the finite user case $(N>2)$. However, we can still extrapolate our two-user results for systems with $N>2$ by orthogonalizing all users into groups of two and implementing optimal scheduling or persistent ALOHA depending on the level of MPR capability available in each group. For example, one could think of separating users based on their spatial locations into groups of two in such a way that no group interferes with another group. This could be achieved by receiver beamforming at the base station or other spatial diversity techniques. Though this technique is suboptimal, it shows how a tradeoff between MAC layer complexity and physical layer complexity can be achieved.

\section{B. Sufficient Condition for the Asymmetric Case, $N>2$}

Deriving stability conditions for the asymmetric $N>2$ case is quite hard even for the collision channel model. Nonetheless, for a fixed transmission probability vector $(p)$, Szpankowski [4] gave a sufficient and necessary condition for stability of the ALOHA system with the collision channel model for the $N>2$ case. In this subsection, we restrict ourselves to finding sufficient conditions for stability for the general reception model for a fixed transmission probability vector $(\boldsymbol{p})$. The main ideas involved here are those of stochastic dominance and of constructing suitable dominating systems for which stability conditions are easier to determine. The way to construct such dominant systems is to assume that some of the queues in the system continue to transmit interfering dummy packets even when they are empty. Because of the dominance, sufficient stability conditions for the dominant system are enough for the original system as well. For the collision channel, such systems are known to stochastically dominate the original ALOHA system [2].

Let $\mathcal{P}=(\mathcal{V}, \mathcal{U})$ be a partition of $\mathcal{M} \triangleq\{1,2, \ldots, N\}$ such that users in $\mathcal{V} \neq \mathcal{M}$ behave just like those in the original ALOHA system while those in $\mathcal{U}$ continue to transmit dummy packets even when their queues are empty. We call users in $\mathcal{U}$ persistent and those in $\mathcal{V}$ nonpersistent. For a partition $\mathcal{P}$ defined above, let $\Theta^{\mathcal{P}}$ denote the ALOHA system where users behave as specified by $\mathcal{P}$. Further, let $\overline{\boldsymbol{Q}}_{\mathcal{P}}^{t}=\left(\overline{\boldsymbol{Q}}_{\mathcal{V}}^{t}, \overline{\boldsymbol{Q}}_{\mathcal{U}}^{t}\right)$ denote the queue lengths in $\Theta^{\mathcal{P}}$.

We note that the marginal reception probabilities given by (2) are not enough to characterize the probability transition matrix for $Q^{t}$. However, we find that the marginal probabilities given by (2) are enough to find sufficient conditions for stability of $\boldsymbol{Q}^{t}$ even for $N>2$. We conjecture that the marginal probabilities of success are sufficient to completely characterize the stability region for a fixed transmission probability. For a slotted ALOHA system with set of (nonpersistent) users $\mathcal{M}$, we denote the set of marginal probabilities of success of all the users by $q^{\mathcal{M}}$. More precisely, if $q_{i \mid \mathcal{S}}^{\mathcal{M}} \triangleq q_{i \mid \mathcal{S}}$, where $q_{i \mid \mathcal{S}}$ is defined by (2) then

$$
\boldsymbol{q}^{\mathcal{M}}=\left\{q_{i \mid \mathcal{S}}^{\mathcal{M}}: i \in \mathcal{S}, \mathcal{S} \subseteq \mathcal{M}\right\} .
$$

We also assume that the reception probabilities $\left(\boldsymbol{q}^{\mathcal{M}}\right)$ permit $\Theta^{\mathcal{P}}$ to stochastically dominate the original system.

The point to note is that the $|\mathcal{V}|<N$-dimensional process $\overline{\boldsymbol{Q}}_{\mathcal{V}}^{t}$ is also a Markov chain which mimics the original ALOHA system [4] except with modified reception probabilities $\left(\boldsymbol{q}^{\mathcal{V}}=\right.$ $\left.\left\{q_{i \mid \mathcal{S}}^{\mathcal{V}}, i \in \mathcal{S}, \mathcal{S} \subseteq \mathcal{V}\right\}\right)$. Thus, we can use induction arguments to establish its stability. More precisely, for any $\mathcal{V}^{\prime} \subseteq \mathcal{V}$ and $i \in$ $\mathcal{V}^{\prime}$, the modified reception probabilities for the smaller ALOHA system consisting of the stand-alone nonpersistent set $\mathcal{V}$ become

$$
q_{i \mid \mathcal{V}^{\prime}}^{\mathcal{V}}=\sum_{\mathcal{T} \subseteq \mathcal{U}}\left(\prod_{j \in \mathcal{T}} p_{j} \prod_{k \in \mathcal{U} \backslash \mathcal{T}} \bar{p}_{k}\right) q_{i \mid \mathcal{V}^{\prime} \cup \mathcal{T}}^{\mathcal{M}}
$$

Now, suppose that the Markov chain $\overline{\boldsymbol{Q}}_{\mathcal{V}}^{t}$ is stationary and ergodic. We denote the stationary version of queue lengths in the nonpersistent set by $\bar{Q}_{\mathcal{V}}$. If we initialize $\bar{Q}_{\mathcal{V}}^{t}$ with its stationary distribution, the departure process from $j$ th users' queue in $\Theta^{\mathcal{P}}$ is also stationary and ergodic. Let $\mathcal{V}=\left\{v_{1}, v_{2}, \ldots, v_{|\mathcal{V}|}\right\}$ and define

$$
\mathcal{X}(\boldsymbol{Q})=\left(\mathbf{1}\left[Q_{1}>0\right], \mathbf{1}\left[Q_{2}>0\right], \ldots, \mathbf{1}\left[Q_{|\boldsymbol{Q}|}>0\right]\right)
$$

where $\mathbf{1}[\cdot]$ is the indicator function. Also, for

$$
z_{\mathcal{V}}=\left(z_{1}, z_{2}, \ldots, z_{|\mathcal{V}|}\right) \in\{0,1\}^{|\mathcal{V}|}
$$

define

$$
\mathcal{C}\left(\mathcal{V}, z_{\mathcal{V}}\right)=\left\{v_{i}: z_{i}=1, i \leq|\mathcal{V}|\right\} .
$$

For $j \in \mathcal{U}$, let $P_{\Theta^{\mathcal{P}}}^{j}$ be the probability of success of the $j$ th user in $\Theta^{\mathcal{P}}$ in the stationary version constructed above. Then, we have

$$
\begin{aligned}
& P_{\Theta^{\mathcal{P}}}^{j}=p_{j}\left[\sum_{\boldsymbol{z}_{\mathcal{V}} \in\{0,1\}^{|\mathcal{V}|}} \operatorname{Pr}\left\{\mathcal{X}\left(\overline{\boldsymbol{Q}}_{\mathcal{V}}\right)=\boldsymbol{z}_{\mathcal{V}}\right\}\right. \\
& \left.\left(\sum_{\mathcal{B} \subseteq \mathcal{U} \cup \mathcal{C}\left(\mathcal{V}, \boldsymbol{z}_{\mathcal{V}}\right) \backslash j} \prod_{k \in \mathcal{B}} p_{k} \prod_{l \in \mathcal{U} \cup \mathcal{C}\left(\mathcal{V}, \boldsymbol{z}_{\mathcal{V}}\right) \backslash(j \cup \mathcal{B})} \bar{p}_{l} q_{j \mid j \cup \mathcal{B}}\right)\right] .
\end{aligned}
$$

Now define a region $\mathcal{R}\left(\boldsymbol{q}^{\mathcal{M}}\right)$ recursively as

$$
\begin{array}{r}
\mathcal{R}\left(\boldsymbol{q}^{\mathcal{M}}\right) \triangleq \bigcup_{\mathcal{P}}\left\{\boldsymbol{\lambda}_{\mathcal{M}}=\left\{\lambda_{i}\right\}_{i \in \mathcal{M}}: \lambda_{k}<P_{\Theta^{\mathcal{P}}}^{k} \forall k \in \mathcal{U},\right. \\
\text { and } \left.\boldsymbol{\lambda}_{\mathcal{V}} \in \mathcal{R}\left(\boldsymbol{q}^{\mathcal{V}}\right)\right\}
\end{array}
$$

with $\mathcal{R}\left(\boldsymbol{q}^{\{i\}}\right) \triangleq\left\{\lambda_{i}<p_{i} q_{i \mid i}^{\{i\}}\right\}$.

Now, we claim the sufficient condition for stability in the form of this theorem.

Theorem 5: Under conditions of stochastic dominance of $\Theta^{\mathcal{P}}$ over the original ALOHA system, if $\lambda_{\mathcal{M}} \in \mathcal{R}\left(\boldsymbol{q}^{\mathcal{M}}\right)$, then the ALOHA system is stable. In other words, $\mathcal{R}\left(\boldsymbol{q}^{\mathcal{M}}\right) \subseteq \mathfrak{S}_{\text {ALOHA }}$.

Proof: Refer to [14].

The reasoning behind why $\boldsymbol{\lambda}_{\mathcal{M}} \in \mathcal{R}\left(\boldsymbol{q}^{\mathcal{M}}\right)$ is sufficient for stability is quite simple; for a particular partition $\mathcal{P}, \boldsymbol{\lambda}_{\mathcal{V}} \in \mathcal{R}\left(\boldsymbol{q}^{\mathcal{V}}\right)$ is sufficient for stability (by induction arguments) of the Markov chain consisting of the nonpersistent set and this makes the departure process for queues in the persistent set stationary and 
ergodic. Then, $\lambda_{k}<P_{\Theta^{\mathcal{P}}}^{k} \forall k \in \mathcal{U}$ is sufficient for stability of persistent queues by Loynes theorem. Thus, $\Theta^{\mathcal{P}}$ is stable and by virtue of stochastic dominance, the original system is stable.

As an example, consider the asymmetric orthogonal channel case. The inner bound for the stability region given by Theorem 5 for this case is actually tight. This follows since for orthogonal channels we have

$$
q_{i \mid \mathcal{S}}^{\mathcal{M}}=q_{i \mid i}^{\{i\}}, \quad \forall i \in \mathcal{S}, \forall \mathcal{S} \subseteq \mathcal{M}
$$

It is easy to check that the reception probabilities satisfying (29) are sufficient for stochastic dominance. Further, it can also be easily seen that the region $\mathcal{R}\left(\boldsymbol{q}^{\mathcal{M}}\right)$ for a fixed transmission probability vector $\boldsymbol{p}$ is given by

$$
\mathcal{R}\left(\boldsymbol{q}^{\mathcal{M}}\right)=\left\{\boldsymbol{\lambda}_{\mathcal{M}}: \lambda_{i}<p_{i} q_{i \mid i}^{\{i\}} \forall i \in \mathcal{M}\right\}
$$

Clearly, $\boldsymbol{p}=\mathbf{1}$ is the best policy in such a case. Similarly, for the case of orthogonal doublets, the sufficient condition given by Theorem 5 is tight since in this case, $\mathcal{R}\left(\boldsymbol{q}^{\{1,2\}}\right)=\mathfrak{S}_{\text {ALOHA }}$ for all the orthogonal doublets. However, the sufficient condition given by Theorem 5 is too difficult if not impossible to evaluate in practice in general. This is because evaluating the stationary distribution of the queues for $N>2$ with arbitrary input distributions in closed form is an unsolved problem as observed in [4], [5], [20], [21].

\section{MPR Through Multiple AntenNAS: An ExAmple}

\section{A. Two-User Case}

To get more insights into the analytical results in Section V, we now apply our results to a two-user scenario to compare different receiver front-ends.

We consider two users, each communicating with a central base station that employs a linear array of $M$ antennas. The two users use slotted ALOHA as the MAC. We assume that the slots are synchronized. The two users are located relatively far away from the base station at fixed angular positions $\boldsymbol{\theta}=$ $\left[\theta_{1}, \theta_{2}\right]$ with respect to the array normal. We assume that most of the energy from user transmissions is received from a planar wavefront arriving at the angle $\boldsymbol{\theta}$. Under these assumptions, we can describe the received signal at the base station $\boldsymbol{y}$ as

$$
\boldsymbol{y}=\boldsymbol{V}(\boldsymbol{\theta}) \boldsymbol{H} \boldsymbol{s}+\boldsymbol{n}
$$

where $\boldsymbol{V}(\boldsymbol{\theta})$ is a Vandermonde matrix of array responses, $\boldsymbol{H}=$ $\operatorname{diag}\left[h_{1}, h_{2}\right]$ is a diagonal matrix of channel (flat) fading for the two users, $\boldsymbol{s}=\left[s_{1}, s_{2}\right]^{T}$ is a vector of users' transmitted symbols, and $\boldsymbol{n} \sim \mathcal{C N}\left(0, I_{M}\right)$ is additive white Gaussian noise.

We also assume slow channel fading that is independent for the two users and i.i.d. from slot to slot. For our numerical results, we assume Rayleigh fading with zero mean and covariance matrix $\Sigma=\operatorname{diag}\left[\sigma_{h_{1}}^{2}, \sigma_{h_{2}}^{2}\right]$. User symbols $\left(s_{i}, i=\right.$ $1,2)$ are independent of each other and the channel fading with $\mathbb{E}\left(\left|s_{i}\right|^{2}\right)=1, i=1,2$. We also assume noncoherent receiver operation, i.e., the base station does not know the channel realization, when it implements the front-end. We consider the effect of coherent receiver operation and knowledge of queue statistics at the base station in [22]. We represent the front-end processing by $\boldsymbol{F}$ (the $i$ th row of $\boldsymbol{F}$ is the set of beamforming weights for the $i$ th user) as follows:

$$
\begin{aligned}
z=F y & =\overbrace{F V(\theta) H}^{R} s+F n \\
& =R s+w .
\end{aligned}
$$

The most important assumption we make is that of the signal-tointerference-plus-noise ratio (SINR) threshold model for packet success, i.e., a packet is successfully received and decoded for user $i$ if

$$
\mathbb{E}\left(\operatorname{SINR}_{i} \mid \boldsymbol{H}\right)=\frac{\left|r_{i i}\right|^{2}}{\left|r_{i \bar{i}}\right|^{2}+\mathbb{E}\left(\left|w_{i}\right|^{2}\right)} \geq \beta
$$

where the expectation is taken over user symbols and noise. In the above, $\bar{i}=\{1,2\} \backslash\{i\}$ and $\beta$ is a threshold which depends on the quality of service requirement. Under the SINR threshold model, the $\boldsymbol{q}$ vector of packet success probabilities for a particular $F$ can be found as

$$
\begin{aligned}
q_{i \mid\{i\}} & =\operatorname{Pr}\left\{\frac{\left|r_{i i}\right|^{2}}{\mathbb{E}\left(\left|w_{i}\right|^{2}\right)}>\beta\right\} \\
q_{i \mid\{1,2\}} & =\operatorname{Pr}\left\{\frac{\left|r_{i i}\right|^{2}}{\left|r_{i \bar{i}}\right|^{2}+\mathbb{E}\left(\left|w_{i}\right|^{2}\right)}>\beta\right\} .
\end{aligned}
$$

The explicit computation of $\boldsymbol{q}$ is provided in [14]. We now consider the performance of three different front-ends for the above system.

1) Decorrelating or Zero-Forcing (ZF): $\boldsymbol{F}$ is the pseudoinverse of $\boldsymbol{V}(\boldsymbol{\theta})$.

2) Matched Filter (MF): $\boldsymbol{F}=\boldsymbol{V}^{H}(\boldsymbol{\theta})$.

3) Pseudo-MMSE (pMMSE): For this receiver,

$$
\boldsymbol{F}=\operatorname{diag}\left[\sigma_{h_{1}}^{2}, \sigma_{h_{2}}^{2}\right] \boldsymbol{V}^{H}(\boldsymbol{\theta}) \boldsymbol{R}_{y y}^{-1}
$$

where $\boldsymbol{R}_{y y}$ is the correlation matrix of $\boldsymbol{y}$ assuming both users transmit. Note that the perfect MMSE receiver needs to know which users are transmitting and the channel realizations in order to find the optimal weights.

Using the stability region as a figure of merit, we can now compare the stability regions of these front-ends in various situations of interest.

1) Symmetric Case: In this case, the channels for the two users are symmetric, i.e., $\sigma_{h_{1}}^{2}=\sigma_{h_{2}}^{2}$. In Fig. 6, we see stability regions for the three different front-ends when the two users are relatively close, $\boldsymbol{\theta}=[54,63]^{\circ}$. We observe that in this rather pessimistic scenario, when one of the users demands a very low rate (close to the axes) the MF performs better than the $\mathrm{ZF}$ and pMMSE. This is not surprising since the MF is optimal if only one user transmits; the ZF suffers from noise enhancement, whereas the pMMSE assumes that both users transmit in every slot. On the other hand, the ZF and pMMSE perform much better than the MF when both users demand an equal rate since in that case both the ZF and pMMSE suppress the interference from the other user better than the MF. We also note that the stability region of the ZF is a rectangle, since the ZF decouples the two users' signals. 


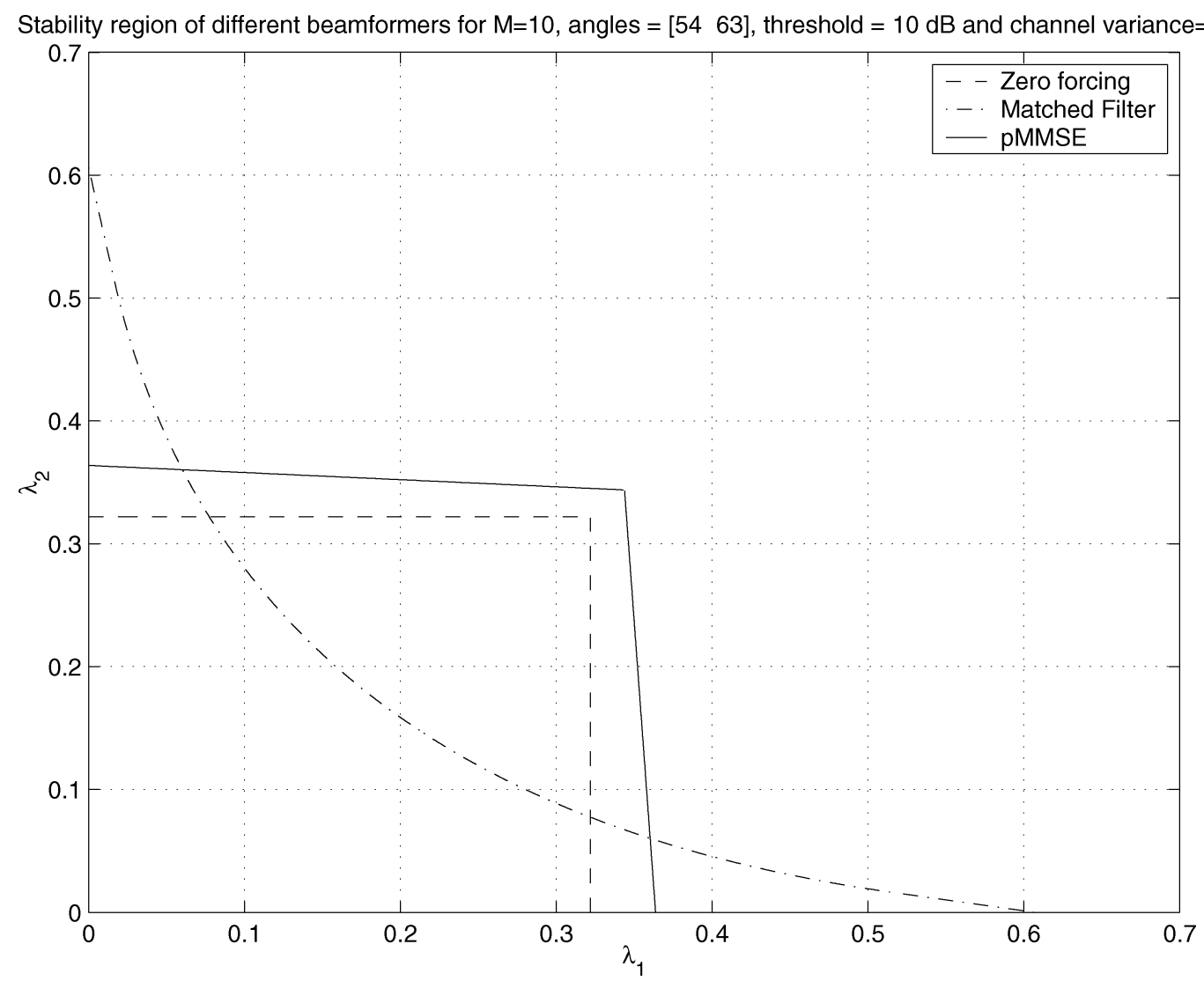

Fig. 6. $M=10, \boldsymbol{\theta}=[54,63]$, threshold $=10 \mathrm{~dB}$, channel $\sim$ gain $=3 \mathrm{~dB}$.

2) Asymmetric Case: Fig. 7 shows the situation when the second user has a very good channel as compared to the first and the users are almost collinear, $\boldsymbol{\theta}=[54,58]^{\circ}$. We see a near-far effect with the ZF and pMMSE front-ends, whereas the MF performs very well. It is not surprising since the MF does not really attempt to null out the other user while the ZF and pMMSE do that. Because of the angular proximity, the ZF and pMMSE suffer. We also note that the stability region of the pMMSE contains the stability region of the ZF receiver in Figs. 6 and 7.

\section{Delay Performance of ALOHA for CAPTURE ChanNels}

Now, we consider characterizing delay in slotted ALOHA systems with multipacket reception. Sidi and Segall [23] analyzed delay in buffered ALOHA type systems and found the exact average delay in a two-user system with symmetric arrival rates and transmission probabilities under a collision channel. They also found optimal transmission probabilities to minimize delay. Further, Nain [24] calculated the exact delay in the twouser case for asymmetric arrivals and transmission probabilities assuming a collision channel. The technique used to find delay involves solving a functional equation in the generating function of the joint stationary queue length distribution. This functional equation can be solved by formulating a Riemann-Hilbert boundary value problem [25], [26]. It is indeed quite surprising that there are no results on the "exact" delay of ALOHA for this queueing model apart from these two. Takagi and Kleinrock [27] use a similar approach to find average delay in a two- user buffered carrier-sense multiple-access/collision detection (CSMA/CD) system with a collision channel. There is also a line of work that computes bounds on average delay for $N>2$ for the collision channel [2], [21], [28], [29], and for a more general symmetric MPR model [9]. There are quite a few other results on delay of ALOHA but they are for different queueing models, viz., infinite user single buffer, finite user single buffer. These models do not quite capture the interdependence among the queues and its effect on delay. The limited results found suggest that characterizing delay in ALOHA systems is a nontrivial task.

In this section, we characterize delay for a subclass of MPR channels, viz., capture channels. In a capture channel, there is a chance that at most one user has a successful packet transmission even if many users transmit in that slot. In some sense, it is an elementary generalization of the collision channel with probabilistic receptions. We focus our attention on the two-user symmetric ALOHA system. We assume that every user has an infinite buffer in which he can store arriving and backlogged packets. The arrivals to the $i$ th user are i.i.d. $\operatorname{Bernoulli}\left(\lambda_{i}\right)$ in every slot. The arrivals are independent across users. The reception model is like the one in the preceding sections.

By definition, for a capture channel, $q_{\{1,2\} \mid\{1,2\}}=0$. Let $q_{1 \mid\{1\}}=q_{2 \mid\{2\}}=a$ and $q_{1 \mid\{1,2\}}=q_{2 \mid\{1,2\}}=b$. Further, we assume $a \geq b$ (to use results of the previous sections). Note that the capture model implies that $a \leq 1$ and $b \leq 0.5$. Also, let $\lambda_{1}=\lambda_{2}=r$ and $p$ be the transmission probability of both users. 


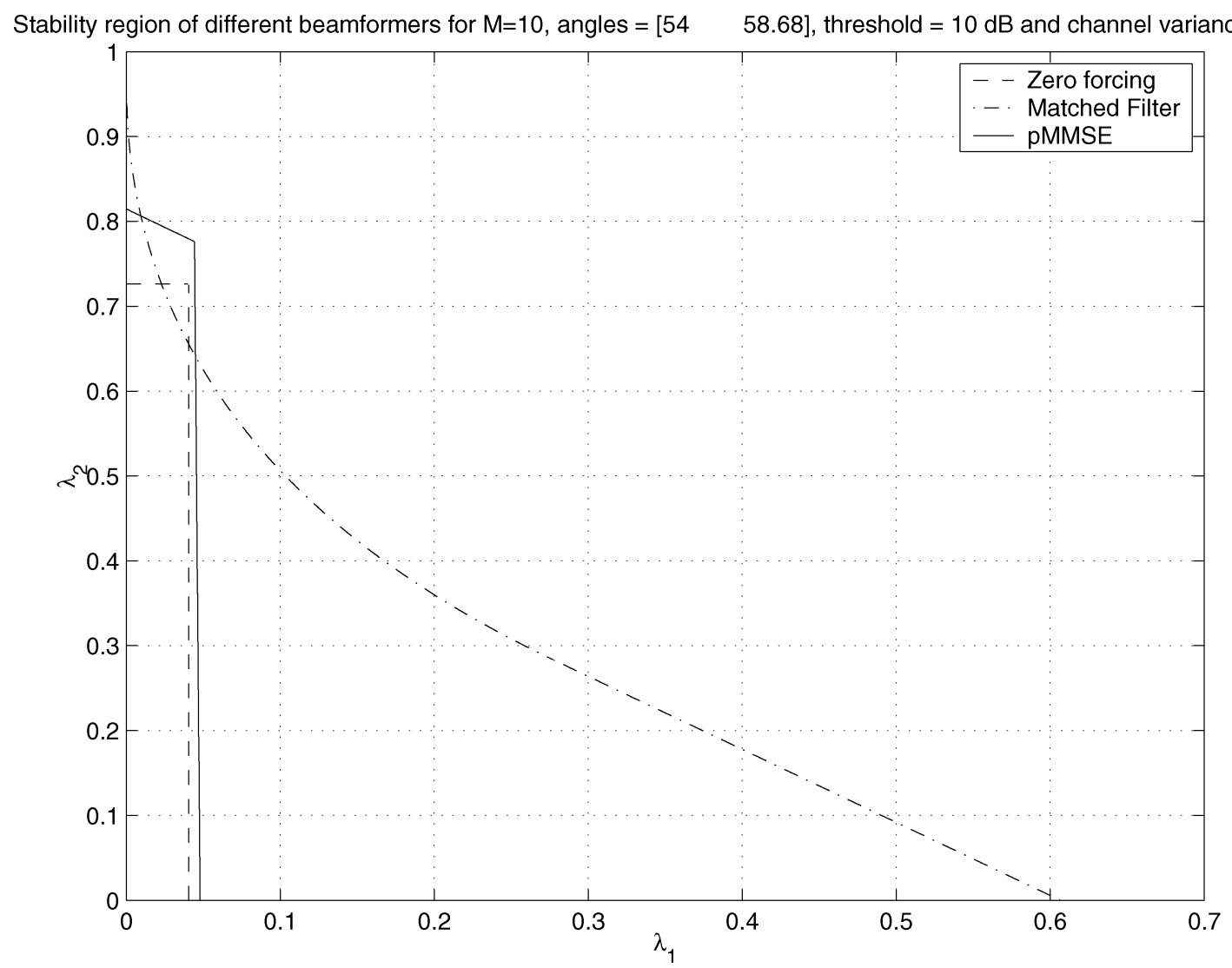

Fig. 7. $M=10, \boldsymbol{\theta}=[54,58]$, threshold $=10 \mathrm{~dB}$, channel gains $=[3,13] \mathrm{dB}$.

Theorem 6: Let $D$ be the average delay for either user in the symmetric capture channel. If the system is stable, i.e., $r<$ $p a+p^{2}(b-a)$

$$
D=\frac{1}{a}\left[\frac{a(1-r)+p(b-a)(1-r / 2)}{p a+p^{2}(b-a)-r}\right] .
$$

Proof: Refer to the Appendix.

From (34), we observe that the delay is an increasing function of $r$, as expected.

Next, we look at the problem of optimizing the transmission probability $\left(p^{*}\right)$ to minimize the average delay. We find that as soon as there is capture capability, the optimal transmission probability is one for a set of arrival rates of the form $\left[0, r^{*}\right]$ with $r^{*}>0$. Thus, persistent ALOHA is delay optimal in the class of ALOHA protocols with fixed transmission probability for small arrival rates.

Lemma 3: Let $p^{*}$ be the optimal transmission probability for minimizing delay in the capture channel. Then,

$p^{*}= \begin{cases}1, & r \in\left[0, r^{*}\right] \\ \frac{a(1-r)-\sqrt{0.5 r} \sqrt{2(a-b)(1-r / 2)^{2}-a^{2}(1-r)}}{(a-b)(1-r / 2)}, & r \in\left(r^{*}, r_{\max }\right)\end{cases}$

where

$$
r^{*}= \begin{cases}\phi(a, b), & 0 \leq b<a / 2 \\ b, & a / 2 \leq b \leq \min \{0.5, a\}\end{cases}
$$

and

$$
r_{\max }= \begin{cases}\frac{a^{2}}{4(a-b)}, & 0 \leq b<a / 2 \\ b, & a / 2 \leq b \leq \min \{0.5, a\} .\end{cases}
$$

In the above

$$
\begin{aligned}
\phi(a, b)=1-\left(\frac{a+b}{2}\right. & \left.-\frac{a b}{a-b}\right) \\
& -\sqrt{1-(a-b)+\left(\frac{a+b}{2}-\frac{a b}{a-b}\right)^{2}} .
\end{aligned}
$$

Proof: Refer to [14].

Lemma 3 gives $p^{*}$ explicitly in terms of the capture channel parameters and the arrival rate $r$. As a direct consequence of Lemma 3 we have the following theorem.

Theorem 7: For the capture channel with $a>0, b>0$, the optimal transmission probabilities can take only two possible forms, as follows.

1) If $b<a / 2$, then the optimal transmission probability is one for a nonempty proper subset of all stable rates of the form $\left[0, r^{*}\right]$ with $0<r^{*}<r_{\max }$.

2) If $b \geq a / 2$, then the optimal transmission probability is one for all stable arrival rates.

Proof: For a fixed $a>0$, from Lemma 3 note that

$$
\left.r^{*}\right|_{b=0}=0 .
$$

It can be shown that $r^{*}$ is a strictly increasing function of $b$ for a fixed value of $a>0$. Thus, as soon as we have capture $(b>0)$, $r^{*}>0$ and there is a set of rates $\left[0, r^{*}\right]$ for which $p^{*}=1$ is the best policy for minimizing delay. As long as $b<a / 2$, we have 

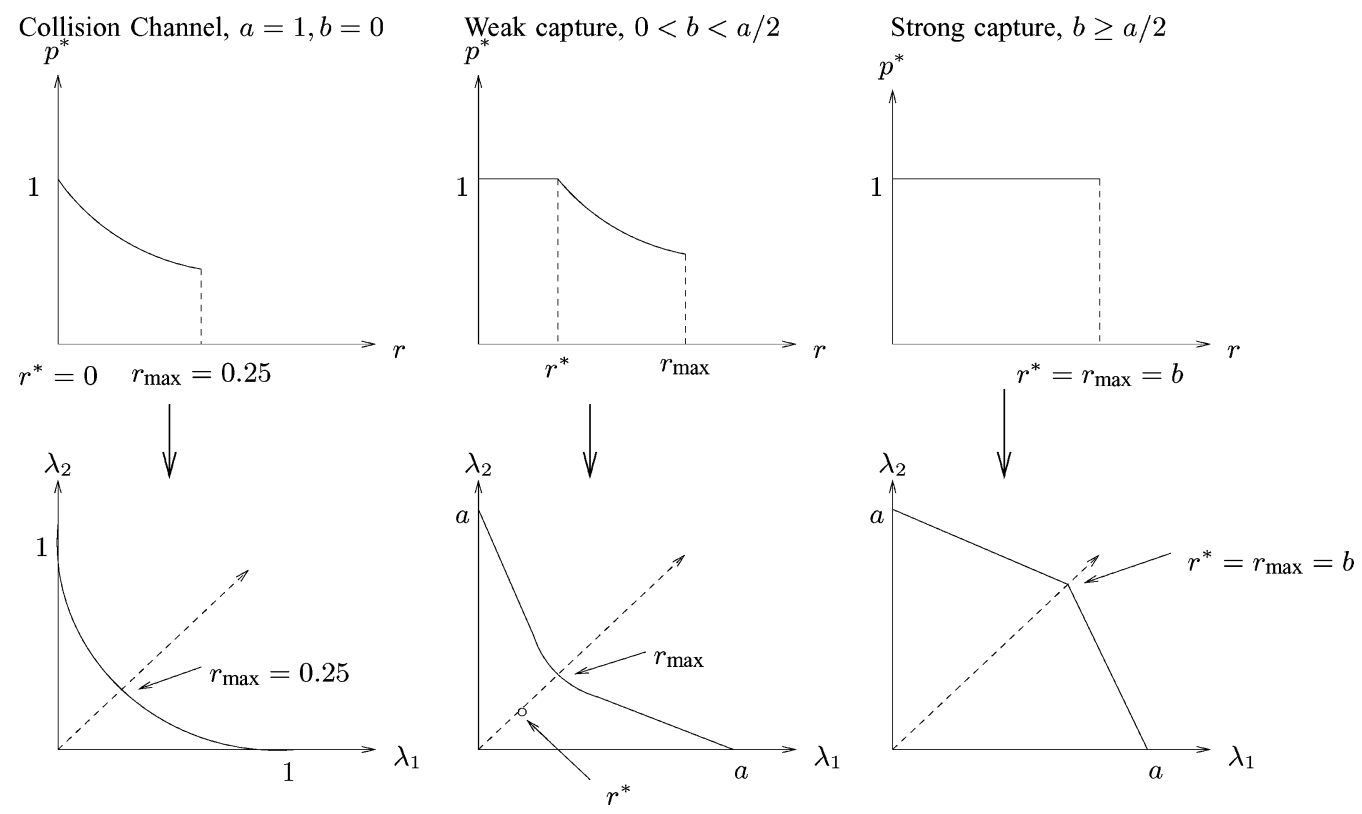

Fig. 8. Generic optimal transmission probabilities for capture channels.

$r^{*}<r_{\max }$. On the other hand, when $b \in[a / 2, \min \{0.5, a\})$, from Lemma 3

$$
\left.\left(r^{*}=r_{\max }\right)\right|_{b \in[a / 2, \min \{0.5, a\})}=b
$$

and so $p^{*}=1$ is delay optimal for any rate which is stabilizable.

Note that for $b<a / 2, r^{*}<r_{\max }$, and there is a set of rates for which the optimal transmission probability $p^{*}$ is still a function of the arrival rate $(r)$. Thus, $b=a / 2$ also happens to be the point where the optimal transmission probability ceases to be a function of the arrival rate. We refer to $r^{*}$ as the critical rate since rates below $r^{*}$ are delay optimized by persistent ALOHA. In Section $\mathrm{V}$, we have already shown that $r_{\max }$ is the maximum stable arrival rate for the capture model.

Fig. 8 shows the generic optimal transmission probabilities as a function of the capture channel parameters. It is interesting to compare the structure of the stability region along the equal rate line with the optimal transmission probability for different capture models. Note that $b=a / 2$ is also the point from which persistent ALOHA is optimal from a stability viewpoint. Thus, persistent ALOHA is optimal from both delay and stability viewpoints when $b \geq a / 2$.

Fig. 9 shows the set of transmission probabilities that stabilize the ALOHA system for different arrival rates in a weak capture $(b<a / 2)$ case. The maximum and minimum stabilizing transmission probabilities are the solution to the equation $p^{2}(b-a)+$ $p a-r=0$ and thus form a parabola which is truncated since the maximum transmission probability can be at most one. The point at which the maximum and minimum transmission probabilities coincide corresponds to the maximum stable arrival rate $r_{\text {max }}$. The delay optimal transmission probability lies in the feasible region in the interior of the parabola.

Now, we look at the delay results in various situations of interest.

Fig. 10 compares the critical rate with maximum stable arrival rate for all possible capture scenarios. We see a phase transition here that occurs at the point $b=a / 2$. As long as $b<a / 2$, persistent ALOHA is only optimal for a subset of the stabilizable rates. On the other hand, as soon as $b \geq a / 2$, persistent ALOHA is optimal for all stabilizable rates. Note that all rates below the solid curve are delay optimized by persistent ALOHA.

\section{A. Delay Comparison of Different Capture Channels}

Fig. 11 compares the minimal delay for three capture scenarios. In this case, we increase $a$ and decrease $b$ progressively. It can be seen that at low arrival rates the capture model with $a=1, b=0.2$ is marginally better than the other capture models. At higher arrival rates, the capture model with $a=0.8$, $b=0.4$ is significantly better than the others. Thus, it seems that for minimizing delay, "multiuser" receiver design is much better than the omnipresent "single-user" designs.

Fig. 12 compares the minimal delay in collision channel $a=$ $1, b=0$ with the delay in strong capture scenarios. It illustrates the significant average delay reduction that can be achieved with the strongest capture model $a=1, b=0.5$. We also note that the minimal delay in this strong capture model $(a=1, b=0.5)$ is quite close to one for arrival rates up to 0.25 . Since the average delay is lower-bounded by one, this suggests that ALOHA is quite close to optimal for a large class of arrival rates for strong capture models.

\section{B. Delay Comparison With Fixed " $a$ "(or " $b$ ")}

Figs. 13 and 14 show minimal delay as a function of the arrival rate for fixed $q_{1 \mid\{1\}}=a$ and fixed $q_{1 \mid\{1,2\}}=b$, respectively. In Fig. 13, the curves are far apart as compared to those in Fig. 14. The figures show that the delay is much more sensitive to changes in $b$ than $a$.

At this point, it is not clear what would happen if we had a stronger reception model than the capture model we have considered in this work. First of all, the technique used to find the average queue length fails as terms corresponding to probability 


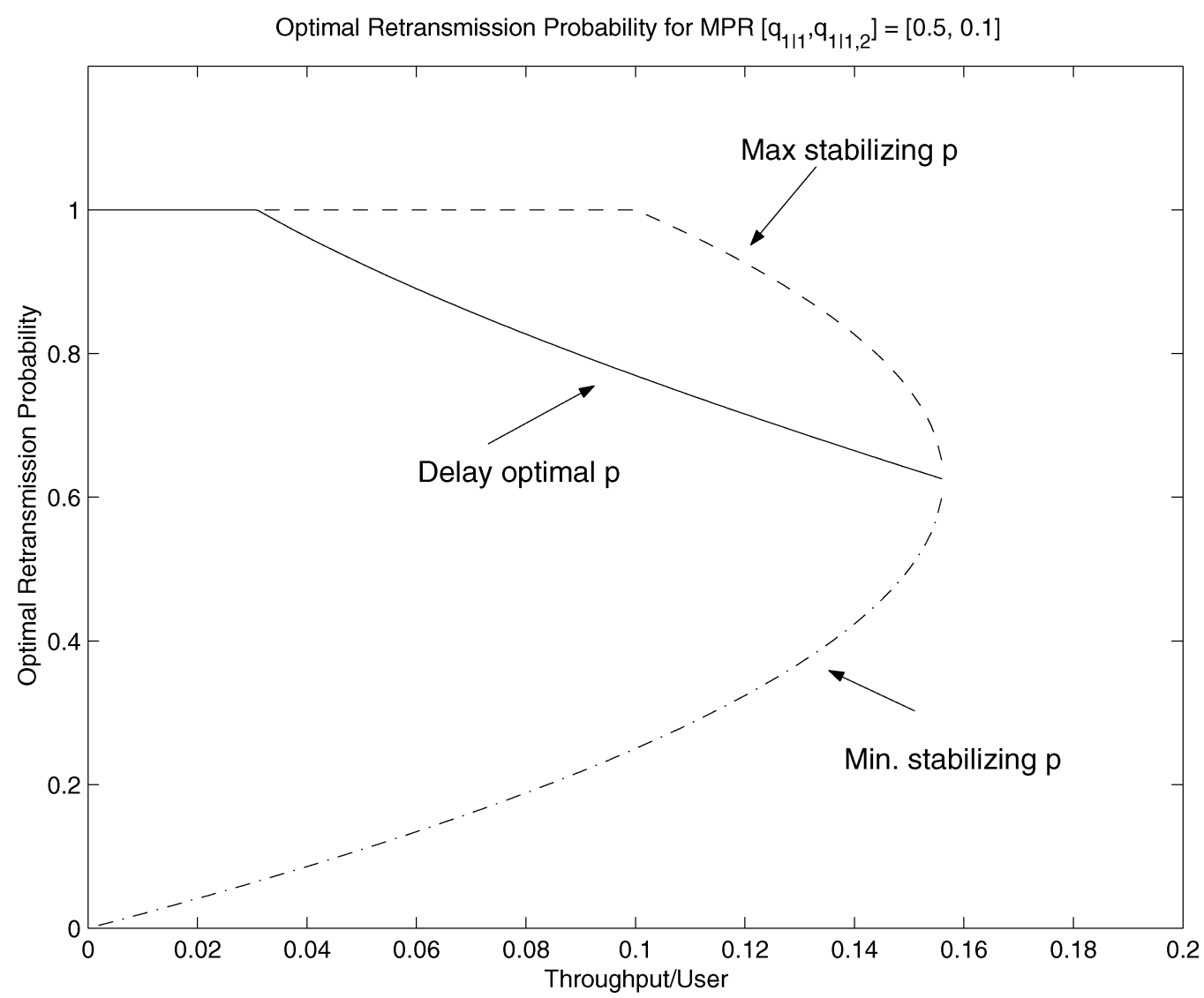

Fig. 9. The optimal and stabilizing transmission probabilities for $a=0.5, b=0.1$.

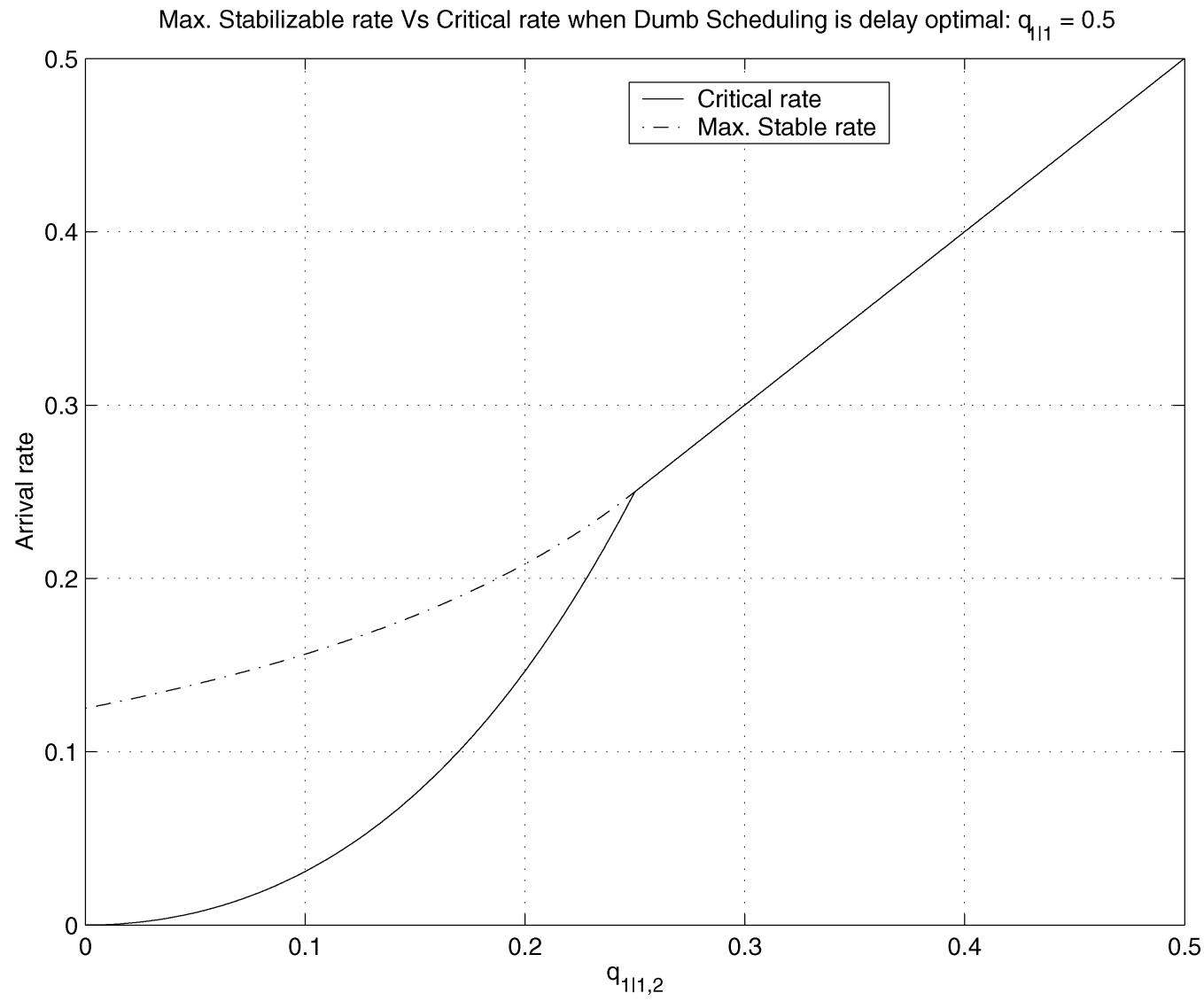

Fig. 10. Maximum stable throughput versus critical rate $q_{1 \mid\{1\}}=a=0.5$. 


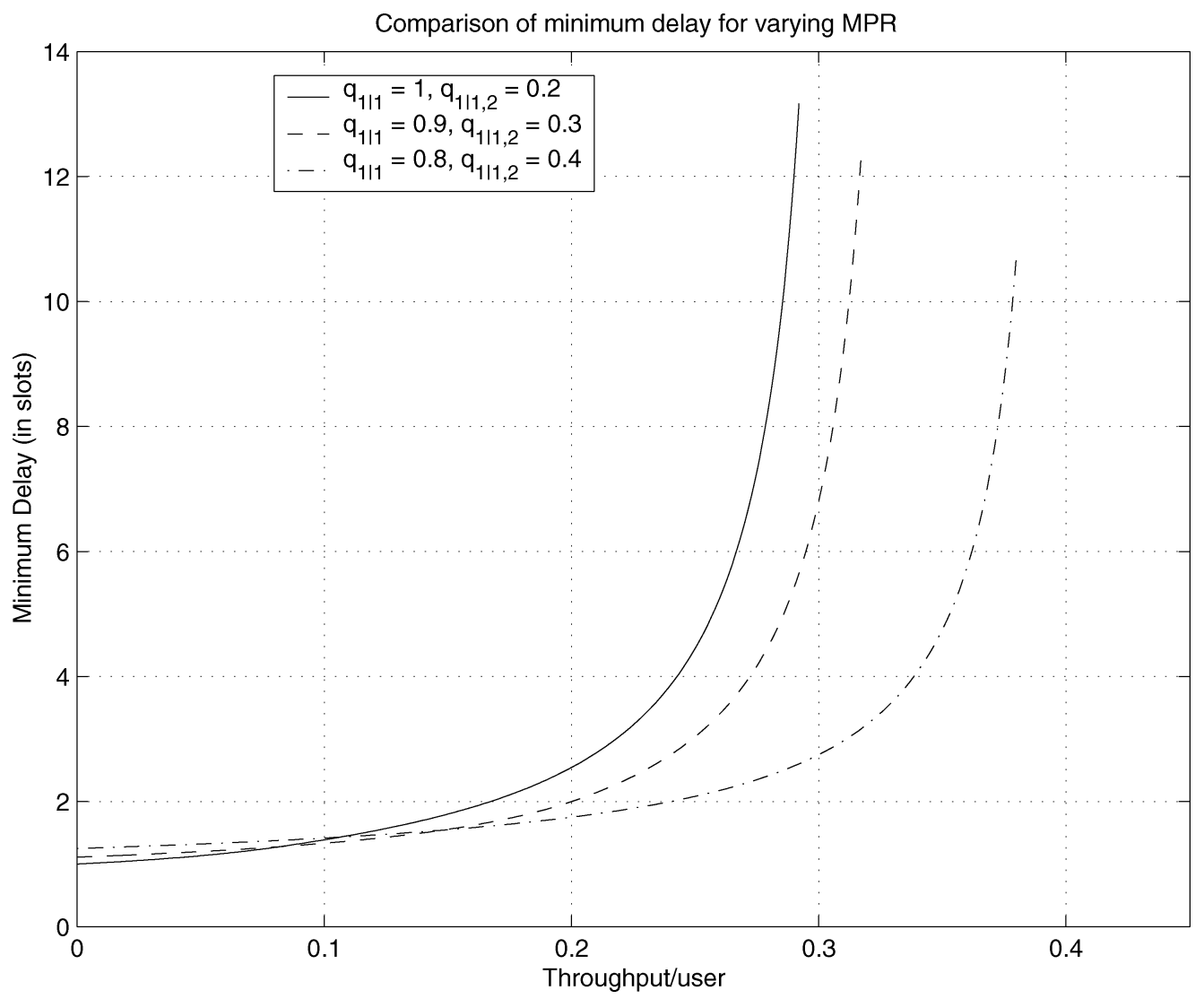

Fig. 11. Comparison of delay for various capture scenarios, $q_{1 \mid\{1\}}=a=1,0.9,0.8, q_{1 \mid\{1,2\}}=b=0.2,0.3,0.4$.

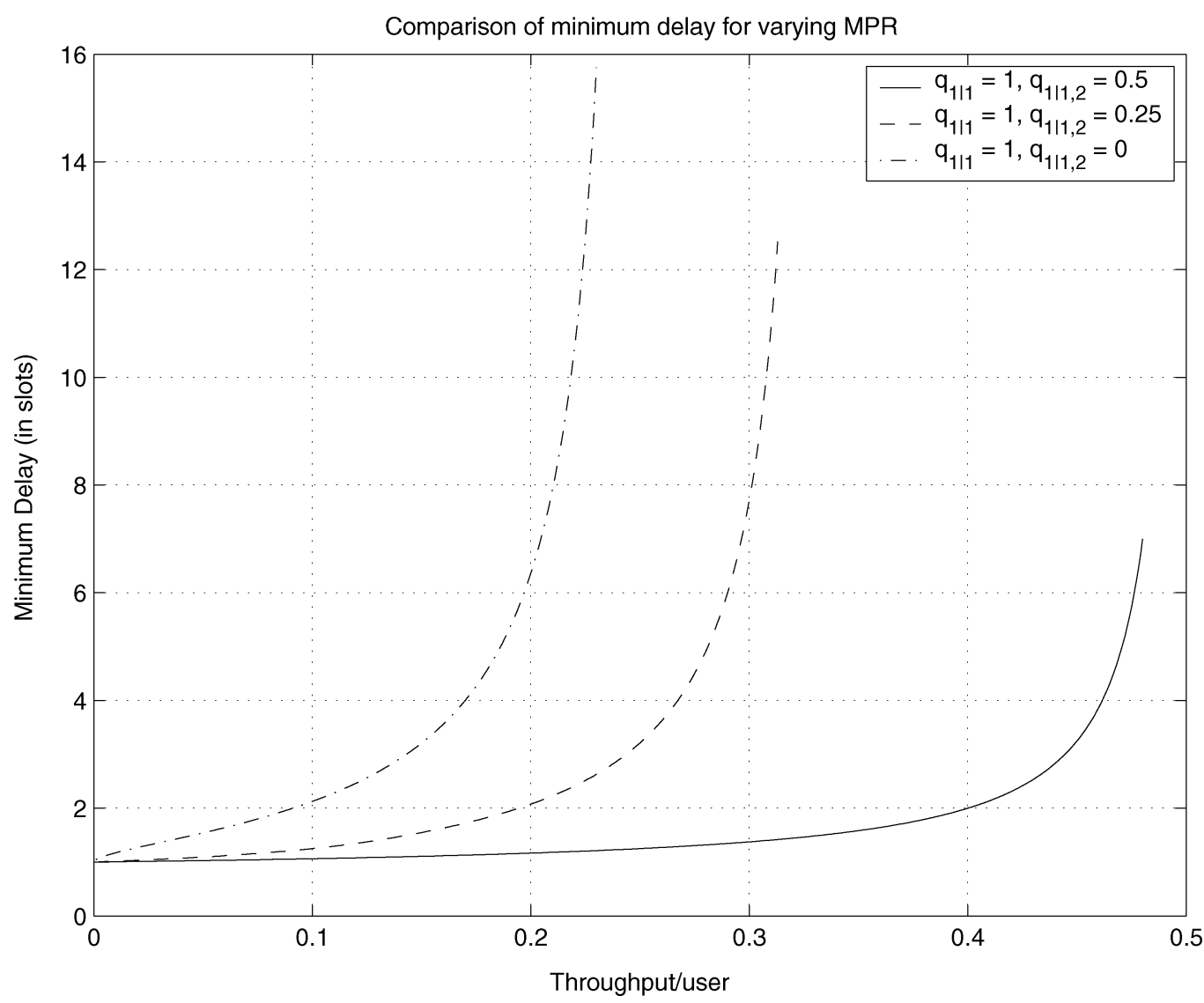

Fig. 12. Comparison of delay for capture scenarios with the collision channel, $q_{1 \mid\{1\}}=a=1,1,1, q_{1 \mid\{1,2\}}=b=0.5,0.25,0$. 


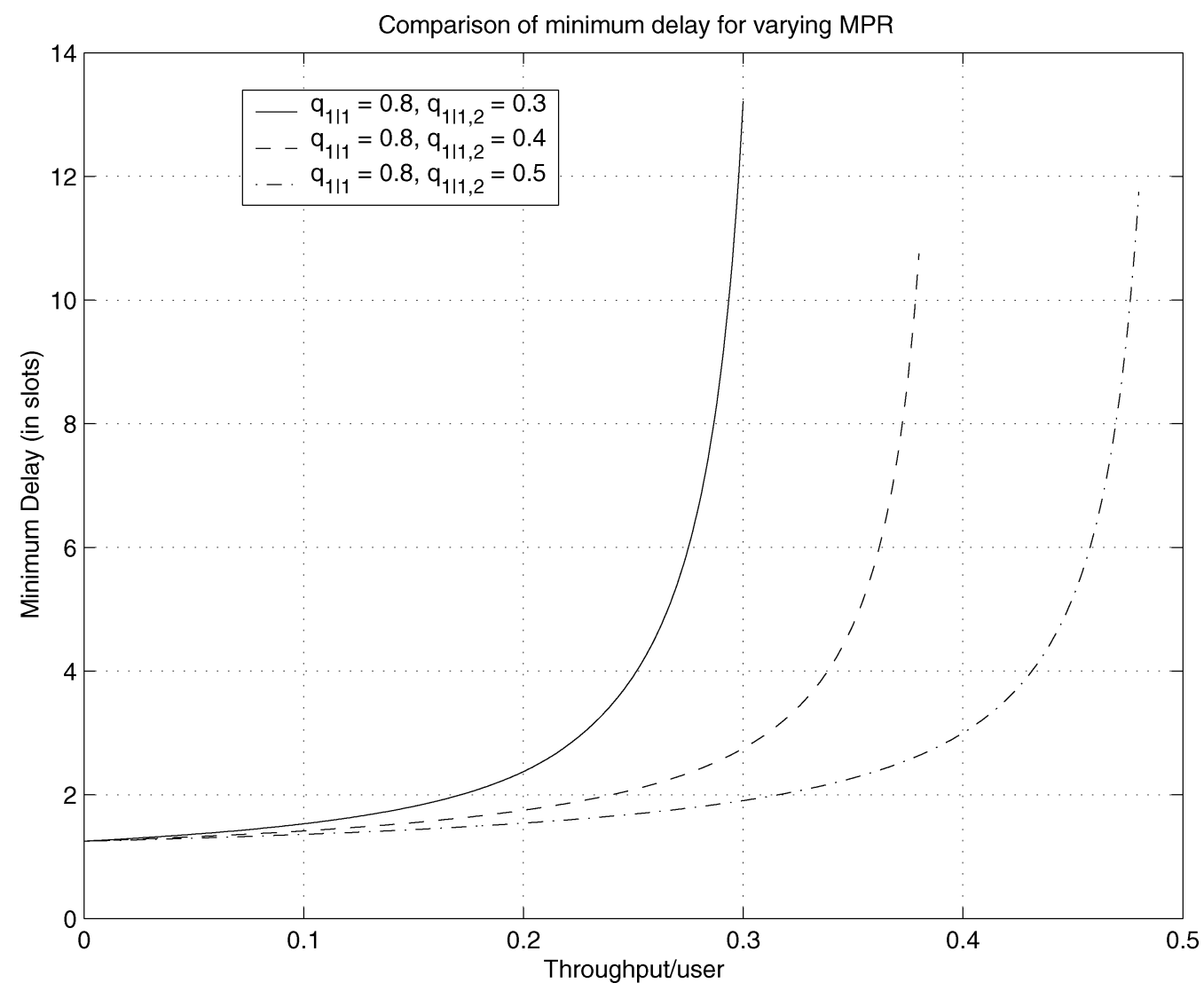

Fig. 13. Comparison of delay for various capture scenarios, $q_{1 \mid\{1\}}=a=0.8,0.8,0.8, q_{1 \mid\{1,2\}}=b=0.3,0.4,0.5$.

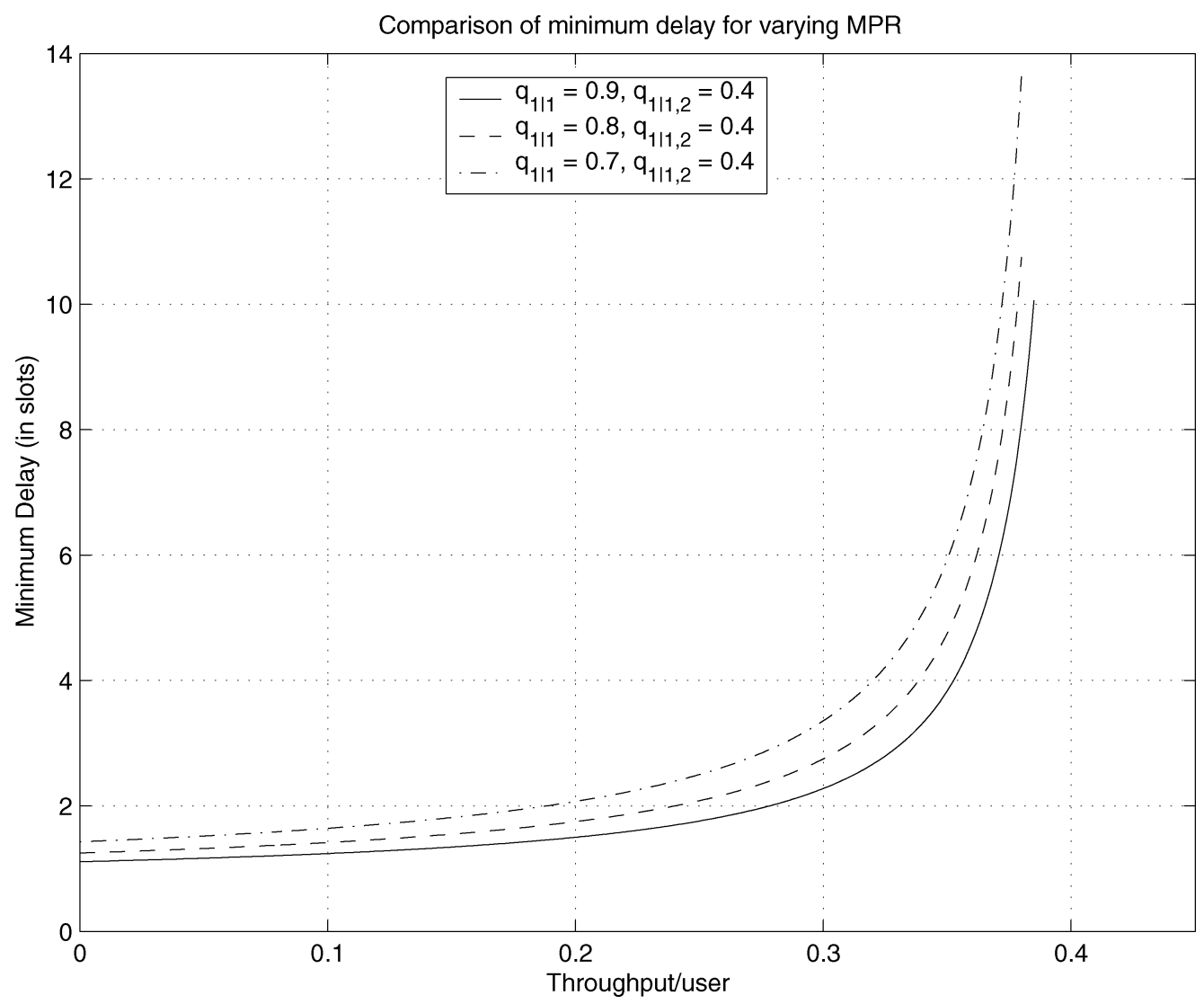

Fig. 14. Comparison of delay for various capture scenarios, $q_{1 \mid\{1\}}=a=0.9,0.8,0.7, q_{1 \mid\{1,2\}}=b=0.4,0.4,0.4$. 
of success of both users simultaneously lead to some complications. However, we conjecture that even with a stronger (symmetric) reception model, i.e., $2 q_{1 \mid\{1,2\}}>1, p=1$ would minimize the delay. The intuition behind this belief is that there is no reason for the users to hold back transmissions in the presence of a stronger reception model.

We also suspect that the delay results that we have will be upper bounds on delay of MPR models which have the same marginal reception probabilities as the capture model we consider, i.e., for MPR models with $q_{1 \mid\{1\}}=a$ and $q_{1 \mid\{1,2\}}=b$ with $q_{\{1,2\} \mid\{1,2\}}>0$.

\section{CONCLUSION}

In this paper, we considered the problem of characterizing stability and delay behavior of slotted ALOHA for multipacket reception models intended to capture the behavior of an improved physical layer. It was shown that the stability region of slotted ALOHA for the two-user case has only four possible forms and that the stability region shows a phase transition from nonconvexity to convexity as the MPR capability increases. After the phase transition, slotted ALOHA was shown to be optimal among all MAC protocols from the point of view of stability. It was also found that when slotted ALOHA is optimal, ALOHA with a transmission probability one (referred to as persistent ALOHA) is optimal. This suggests that after a certain level of MPR capability is provided by the physical layer, MAC layer design is simple; no transmission control is the best policy. Further, it was shown that persistent ALOHA is also optimal for the $N>2$ case for the symmetric MPR model in a wide range of MPR regimes. Generalizing the stability region results to the finite user case seems a rather nontrivial task and although we have been able to provide some sufficient conditions for stability, they are difficult to check. Exact expressions for delay-minimizing transmission probabilities and the exact average delay of slotted ALOHA for a subclass of MPR models (capture channel) for the two-user case were provided. It was also shown that persistent ALOHA is optimal from a delay and stability viewpoint for all stable arrival rates in a certain capture regime and that persistent ALOHA is always delay optimal for a subset of stable arrival rates once capture sets in. Our results present a clear case for the so-called "cross-layer" approach (where a combination of layers (here physical (PHY) and MAC) are designed jointly to optimize network performance) by quantifying the gains that can be achieved through an optimal design of the MAC based on an accurate model of the physical layer.

\section{APPENDIX}

\section{A. Proof of Lemma 1}

We construct parallel dominant ALOHA systems in which one of the queues continues to transmit dummy packets even when the queue is empty. Dummy packets cause interference but the successful reception of a dummy packet has no significance. It has been shown [3] that for the collision channel model, these dominant systems stochastically dominate ${ }^{4}$ the original

\footnotetext{
${ }^{4} \mathrm{~A}$ real random variable $X$ is said to stochastically dominate a real random variable $Y$ if $\forall z \in \mathbb{R}, \operatorname{Pr}\{X>z\} \geq \operatorname{Pr}\{Y>z\}$. We denote this dominance by $X \geq_{s t} Y$.
}

ALOHA system in the sense that if both the dominant system and the original system have the same initial queue sizes, both systems have the same arrivals in every slot, and have the same "coin tossing" outcomes (that determine transmission attempts) in every slot, then the queue sizes in the dominant system are necessarily not smaller than those in the original system. As a result, conditions for stability of dominant systems are sufficient for stability of the original system.

Consider a two-user ALOHA system in which queue 1 transmits dummy packets when it is empty. The probability of success for a packet from queue 2 is always $p_{2} \bar{p}_{1} q_{2 \mid\{2\}}+p_{1} p_{2} q_{2 \mid\{1,2\}}$. However, in the original ALOHA system, the probability of success in queue 2 would be $p_{2} \bar{p}_{1} q_{2 \mid\{2\}}+p_{1} p_{2} q_{2 \mid\{1,2\}}$ if queue 1 were nonempty and $p_{2} q_{2 \mid\{2\}}$ otherwise. If

$$
p_{2} q_{2 \mid\{2\}} \geq p_{2} \bar{p}_{1} q_{2 \mid\{2\}}+p_{1} p_{2} q_{2 \mid\{1,2\}} \Longleftrightarrow Q_{2} \geq 0
$$

the probability of success in queue 2 in the "dummy" packet transmitting system would always be lower than that in the original system. Now consider queue 1. Using standard G/G/1 queue results, it follows that the average probability of success seen in queue 1 is

$$
\begin{aligned}
& p_{1} q_{1 \mid\{1\}}\left(1-\frac{\lambda_{2}}{p_{2} \bar{p}_{1} q_{2 \mid\{2\}}+p_{1} p_{2} q_{2 \mid\{1,2\}}}\right) \\
& +\left(p_{1} \bar{p}_{2} q_{1 \mid\{1\}}+p_{1} p_{2} q_{1 \mid\{1,2\}}\right)\left(\frac{\lambda_{2}}{p_{2} \bar{p}_{1} q_{2 \mid\{2\}}+p_{1} p_{2} q_{2 \mid\{1,2\}}}\right)
\end{aligned}
$$

which simplifies to

$$
p_{1} q_{1 \mid\{1\}}-\frac{p_{1} p_{2} \lambda_{2} Q_{1}}{\lambda_{2}^{*}} .
$$

Now, if

$$
p_{1} q_{1 \mid\{1\}} \geq p_{1} \bar{p}_{2} q_{1 \mid\{1\}}+p_{1} p_{2} q_{1 \mid\{1,2\}} \Longleftrightarrow Q_{1} \geq 0
$$

queue 1 in the "dummy" packet system also stochastically dominates queue 1 in the original system. This is because if (41) is true, then the expression in (40) would certainly be less than the average probability of success in queue 1 in the original ALOHA system.

Now, by definition $\lambda_{2}^{*}=p_{2} \bar{p}_{1} q_{2 \mid\{2\}}+p_{1} p_{2} q_{2 \mid\{1,2\}}$ and so, by a simple application of Loynes theorem, we find that the dummy packet system is stable (with the possible exception of boundary points) if and only if

$$
\lambda_{1} \leq p_{1} q_{1 \mid\{1\}}-\frac{p_{1} p_{2} \lambda_{2} Q_{1}}{\lambda_{2}^{*}}, \quad \lambda_{2} \leq \lambda_{2}^{*} .
$$

If in addition $Q_{1} \geq 0$ and $Q_{2} \geq 0$, by stochastic dominance the original ALOHA system is also stable (see Fig. 15).

By a parallel argument for a system in which queue 2 transmits dummy packets, we find that the original ALOHA system is stable (with the possible exception of boundary points) if $Q_{1} \geq 0$ and $Q_{2} \geq 0$ and

$$
\lambda_{2} \leq p_{2} q_{2 \mid\{2\}}-\frac{p_{1} p_{2} \lambda_{1} Q_{2}}{\lambda_{1}^{*}}, \quad \lambda_{1} \leq \lambda_{1}^{*} .
$$

Combining the sufficient conditions for both the dominant systems gives the sufficiency part of the lemma. 


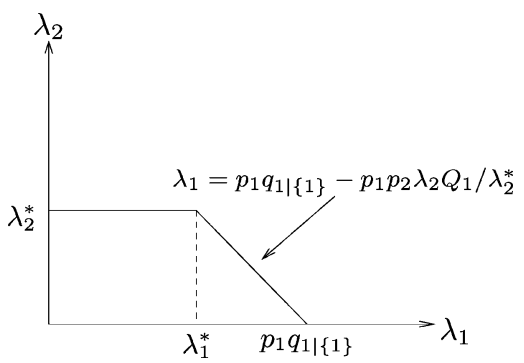

Fig. 15. Stability region of dominant system in which queue 1 transmits dummy packets.

The necessary part of the lemma follows by an "indistinguishability" argument similar to the one used by Rao and Ephremides [3]. Consider the system in which queue 1 transmits dummy packets. If along some realizations of queue 1 of nonzero probability, queue 1 never empties, then the original system and the dummy packet transmitting system are "indistinguishable." Thus, with a particular initial condition, if queue 1 never empties with nonzero probability in the dominant system (which is equivalent to the Markov chain being unstable), then queue 1 in the original system must be unstable as well. Thus, for $\lambda_{2} \leq \lambda_{2}^{*}, \lambda_{1}=p_{1} q_{1 \mid\{1\}}-\frac{p_{1} p_{2} \lambda_{2} Q_{1}}{\lambda_{2}^{*}}$ is the boundary of the stability region of the original system as well. It follows that the conditions given in Lemma 1 are necessary for stability as well.

\section{B. Proof of Lemma 2}

We use Lemma 1. Since we know the stability region for a fixed transmission probability vector $p$, we need to find the union of all the stability regions as the parameter $\boldsymbol{p}$ varies over $[0,1]^{2}$. One way of doing this is to set up a corresponding constraint optimization problem, i.e., for a fixed $\lambda_{1}$, maximize $\lambda_{2}$ as $p$ varies over $[0,1]^{2}$, where $\lambda_{1}$ and $\lambda_{2}$ are related by (10) and (11). The difficulty in using known optimization techniques is that the objective function is not differentiable at a point in its domain and so the optimization has to be carried out rather explicitly.

Replacing $\lambda_{1}$ by $x$ and $\lambda_{2}$ by $y$, we write the boundary given by (10) and (11) as

$$
\begin{aligned}
& x=p_{1} q_{1 \mid\{1\}}-\frac{p_{1} y Q_{1}}{\left(q_{2 \mid\{2\}}-p_{1} Q_{2}\right)}, \\
& \quad \text { for } 0 \leq y<p_{2}\left(q_{2 \mid\{2\}}-p_{1} Q_{2}\right)
\end{aligned}
$$

and

$$
\begin{aligned}
& y=p_{2} q_{2 \mid\{2\}}-\frac{p_{2} x Q_{2}}{\left(q_{1 \mid\{1\}}-p_{2} Q_{1}\right)}, \\
& \quad \text { for } 0 \leq x<p_{1}\left(q_{1 \mid\{1\}}-p_{2} Q_{1}\right) .
\end{aligned}
$$

Note that any $\left(x^{*}, y^{*}\right)$ which satisfies (44) does not satisfy (45) and vice versa.
Now, we consider the following three cases.

- Case 1: $Q_{1}>0$ and $Q_{2}>0$.

Consider the following constrained maximization problem where the domain of $x$ is unconstrained (i.e., $x \geq 0$ )

$$
\max _{p_{2} \in[0,1]} y^{\prime}=\max _{p_{2} \in[0,1]}\left(p_{2} q_{2 \mid\{2\}}-\frac{p_{2} x Q_{2}}{\left(q_{1 \mid\{1\}}-p_{2} Q_{1}\right)}\right) .
$$

Differentiating with respect to $p_{2}$

$$
\frac{d y^{\prime}}{d p_{2}}=q_{2 \mid\{2\}}-\frac{x Q_{2} q_{1 \mid\{1\}}}{\left(q_{1 \mid\{1\}}-p_{2} Q_{1}\right)^{2}} .
$$

Equating the derivative to zero gives $p_{2}^{*}$ as

$$
\left(q_{1 \mid\{1\}}-p_{2}^{*} Q_{1}\right)=\sqrt{\frac{x Q_{2} q_{1 \mid\{1\}}}{q_{2 \mid\{2\}}}} .
$$

A simple check on the second derivative shows that the maximizing $p_{2}^{*}$ is

$$
p_{2}^{*}=\frac{q_{1 \mid\{1\}}}{Q_{1}}-\frac{1}{Q_{1}} \sqrt{\frac{x Q_{2} q_{1 \mid\{1\}}}{q_{2 \mid\{2\}}}} .
$$

But since $p_{2}^{*}$ is a probability, $0 \leq p_{2}^{*} \leq 1$. Also, by (48)

$$
\begin{aligned}
0 & \leq p_{2}^{*} \leq 1 \\
& \Longleftrightarrow \frac{q_{2 \mid\{2\}}\left(q_{1 \mid\{1\}}-Q_{1}\right)^{2}}{q_{1 \mid\{1\}} Q_{2}} \leq x \leq \frac{q_{1 \mid\{1\}} q_{2 \mid\{2\}}}{Q_{2}}
\end{aligned}
$$

and so for $x$ in the range given by (50), we have by substituting (49) in (45)

$$
\begin{array}{r}
y_{\max }^{\prime}=\left(\frac{q_{1 \mid\{1\}} q_{2 \mid\{2\}}-\sqrt{x Q_{2} q_{1 \mid\{1\}} q_{2 \mid\{2\}}}}{Q_{1}}\right) \\
\times\left(1-\sqrt{\frac{x Q_{2}}{q_{1 \mid\{1\}} q_{2 \mid\{2\}}}}\right) .
\end{array}
$$

Now, we consider the constraint on the domain of $x$ as given by (45). For a fixed $x,(45)$ is valid only for $p_{2}<$ $\frac{\left(q_{1 \mid\{1\}}-x\right)}{Q_{1}}$. So, we need to show that $p_{2}^{*}$ given by (49) is, in fact, less than $\frac{1}{Q_{1}}\left(q_{1 \mid\{1\}}-x\right)$. Clearly

$$
p_{2}^{*}<\frac{\left(q_{1 \mid\{1\}}-x\right)}{Q_{1}} \text { iff } x<\frac{q_{1 \mid\{1\}} Q_{2}}{q_{2 \mid\{2\}}}
$$

and, therefore, $y_{\max }^{\prime}$ given by (51) is actually really valid only in the range of $x$ given by the intersection of (52) and (50). Now observe that for

$$
\begin{aligned}
0 \leq x & <\frac{q_{2 \mid\{2\}}\left(q_{1 \mid\{1\}}-Q_{1}\right)^{2}}{q_{1 \mid\{1\}} Q_{2}} \\
& \frac{d y^{\prime}}{d p_{2}}>0, \quad \forall p_{2} \in[0,1] .
\end{aligned}
$$




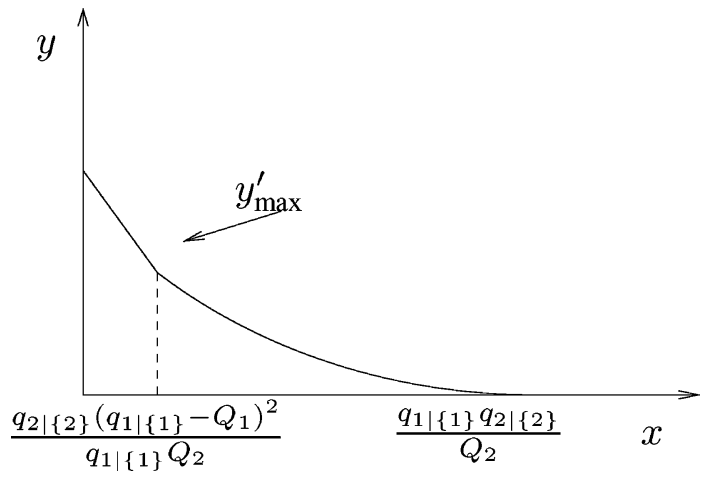

Fig. 16. The appearance of $y_{\max }^{\prime}$ as a function of $x$.

This means that $y^{\prime}$ is a strictly increasing function of $p_{2}$ in the range of $x$ given by (53). Thus, the optimizing $p_{2}^{*}$ in this range of $x$ is equal to one. So, we have

$$
y_{\max }^{\prime}=q_{2 \mid\{2\}}-\frac{x Q_{2}}{\left(q_{1 \mid\{1\}}-Q_{1}\right)} .
$$

But again we have to check that (45) is really valid. Note that for $x<\left(q_{1 \mid\{1\}}-Q_{1}\right), y$ given by (45) is valid for any $p_{2} \in[0,1]$. So, $y_{\max }^{\prime}$ given by (54) is also really valid only in

$$
x<\min \left\{\frac{q_{2 \mid\{2\}}\left(q_{1 \mid\{1\}}-Q_{1}\right)^{2}}{q_{1 \mid\{1\}} Q_{2}},\left(q_{1 \mid\{1\}}-Q_{1}\right)\right\} .
$$

Fig. 16 shows the behavior of $y_{\text {max }}^{\prime}$ as a function of $x$ as given by (51) and (54).

By an exactly parallel argument applied to (44), we can show that

$$
\begin{aligned}
& x_{\max }^{\prime}=q_{1 \mid\{1\}}-\frac{y Q_{1}}{\left(q_{2 \mid\{2\}}-Q_{2}\right)}, \\
& \text { for } 0 \leq y<\frac{q_{1 \mid\{1\}}\left(q_{2 \mid\{2\}}-Q_{2}\right)^{2}}{q_{2 \mid\{2\}} Q_{1}}
\end{aligned}
$$

and

$$
\begin{array}{r}
x_{\max }^{\prime}=\left(\frac{q_{1 \mid\{1\}} q_{2 \mid\{2\}}-\sqrt{y Q_{1} q_{1 \mid\{1\}} q_{2 \mid\{2\}}}}{Q_{2}}\right) \\
\times\left(1-\sqrt{\frac{y Q_{1}}{q_{1 \mid\{1\}} q_{2 \mid\{2\}}}}\right)
\end{array}
$$

for

$$
\frac{q_{1 \mid\{1\}}\left(q_{2 \mid\{2\}}-Q_{2}\right)^{2}}{q_{2 \mid\{2\}} Q_{1}} \leq y \leq \frac{q_{1 \mid\{1\}} q_{2 \mid\{2\}}}{Q_{1}} .
$$

The idea of the proof is as follows: First, we assume that a rate $x$ can be stabilized for the first user and then we find the highest rate for the second user $y_{\max }$ for which the second queue is stable. We also note from the preceding discussion that if

$$
\left(q_{1 \mid\{1\}}-Q_{1}\right)<\frac{q_{1 \mid\{1\}} Q_{2}}{q_{2 \mid\{2\}}}
$$

then

$$
y_{\max }=y_{\max }^{\prime}, \quad \text { for } 0 \leq x<\frac{q_{1 \mid\{1\}} Q_{2}}{q_{2 \mid\{2\}}}
$$

where $y_{\max }^{\prime}$ is given by (54) for

$$
x \in\left[0, \frac{q_{2 \mid\{2\}}\left(q_{1 \mid\{1\}}-Q_{1}\right)^{2}}{q_{1 \mid\{1\}} Q_{2}}\right)
$$

and (51) for

$$
x \in\left[\frac{q_{2 \mid\{2\}}\left(q_{1 \mid\{1\}}-Q_{1}\right)^{2}}{q_{1 \mid\{1\}} Q_{2}}, \frac{q_{1 \mid\{1\}} Q_{2}}{q_{2 \mid\{2\}}}\right] .
$$

On the other hand, if

$$
\left(q_{1 \mid\{1\}}-Q_{1}\right) \geq \frac{q_{1 \mid\{1\}} Q_{2}}{q_{2 \mid\{2\}}}
$$

then $y_{\max }=y_{\max }^{\prime}$ for $0 \leq x<\left(q_{1 \mid\{1\}}-Q_{1}\right)$, where $y_{\max }^{\prime}$ is given by (54). But the problem is that the first users' rate $x$ might, in fact, not be stabilizable. So, we interchange the users and find the best possible rate for the first user assuming the second users' rate can be stabilized. It can be checked that the point where $y_{\text {max }}^{\prime}$ ceases to be equal to $y_{\max }$ is exactly the point from where $x_{\max }^{\prime}=x_{\max }$ and vice versa. Therefore, it is clear that the intersection of the regions below $y_{\max }=f\left(x, q_{1 \mid\{1\}}, q_{2 \mid\{2\}}, Q_{1}, Q_{2}\right)$ and $x_{\max }=f\left(y, q_{2 \mid\{2\}}, q_{1 \mid\{1\}}, Q_{2}, Q_{1}\right)$, where $f$ is defined in (16), is actually the stability region that we seek, because for any rate pair in this intersection, there exists a $\left[p_{1}, p_{2}\right]$ such that the system is stable.

- Case 2: $Q_{1}=0$ and $Q_{2}=0$.

It is obvious from (44) and (45) that in this case the stability region is the rectangle $0 \leq x<q_{1 \mid\{1\}}$ and $0 \leq$ $y<q_{2 \mid\{2\}}$.

- Case 3: $Q_{1}>0$ and $Q_{2}=0$.

For this case, (44) and (45) take the form

$$
y=p_{2} q_{2 \mid\{2\}}, \quad \text { for } 0 \leq x \leq p_{1}\left(q_{1 \mid\{1\}}-p_{2} Q_{1}\right)
$$

and

$$
x=p_{1} q_{1 \mid\{1\}}-\frac{p_{1} y Q_{1}}{q_{2 \mid\{2\}}}, \quad \text { for } 0 \leq y<p_{2} q_{2 \mid\{2\}} .
$$

Now, if $y<q_{1 \mid\{1\}} q_{2 \mid\{2\}} / Q_{1}$ (the only case of interest), then $p_{1}^{*}=1$ maximizes $x$ in (58) and in this range

$$
x_{\max }=q_{1 \mid\{1\}}-\frac{y Q_{1}}{q_{2 \mid\{2\}}} .
$$

Also, $y$ in (57) has a maximum value of $q_{2 \mid\{2\}}$. Again, by arguments similar to Case 1 , the stability region is the region bounded by the lines $y=q_{2 \mid\{2\}}$ and $x=q_{1 \mid\{1\}}-$ $y Q_{1} / q_{2 \mid\{2\}}$.

The case when $Q_{1}=0$ and $Q_{2}>0$ can be handled similarly.

An equivalent formulation would be to find the geometric envelope of the two-parameter $\left(p_{1}, p_{2}\right)$ family of curves which define the boundary of the stability region given in Lemma 1. 


\section{Proof of Theorem 3}

We proceed to prove equivalence between the five statements as follows.

\section{Proof That Part 2) Is Equivalent to Part 3):}

a) $\mathfrak{S}_{\mathrm{ALOHA}}$ is polyhedral $\Longrightarrow(18)$ : If $Q_{1}=0$ and $Q_{2}=$ 0 , then it can be easily seen that the stability region is bounded by perpendicular lines and (18) is trivially satisfied. If either $Q_{1}$ or $Q_{2}$ is equal to zero, then by Case 3 of the proof of Lemma 2, the stability region is polyhedral and (18) holds. So, we consider the case $Q_{1}>0$ and $Q_{2}>0$. Under our assumptions, the point of intersection of the lines defined by (54) and (55) is $\left(q_{1 \mid\{1\}}-\right.$ $\left.Q_{1}, q_{2 \mid\{2\}}-Q_{2}\right)$. From (54) and (55), it follows that the stability region is polyhedral if

$$
\left(q_{1 \mid\{1\}}-Q_{1}\right) \leq \frac{q_{2 \mid\{2\}}\left(q_{1 \mid\{1\}}-Q_{1}\right)^{2}}{q_{1 \mid\{1\}} Q_{2}}
$$

and

$$
\left(q_{2 \mid\{2\}}-Q_{2}\right) \leq \frac{q_{1 \mid\{1\}}\left(q_{2 \mid\{2\}}-Q_{2}\right)^{2}}{q_{2 \mid\{2\}} Q_{1}}
$$

which is equivalent to (18).

b) Equation (18) $\Longrightarrow \mathfrak{S}_{\mathrm{ALOHA}}$ is polyhedral: For the case $Q_{1}=0, Q_{2}=0$, and $Q_{1}$ or $Q_{2}$ equal to zero, we know that the stability region is indeed polyhedral. When $Q_{1}>$ 0 and $Q_{2}>0$, then (18) clearly implies (60) and (61) and these equations in turn imply that the stability region is polyhedral.

Proof of That Part 1) Is Equivalent to Part 2):

a) $\mathfrak{S}_{\mathrm{ALOHA}}$ is polyhedral $\Longrightarrow \mathfrak{S}_{\mathrm{ALOHA}}$ is convex: Consider the line with $\mathrm{X}$-intercept $q_{1 \mid\{1\}}$ and $\mathrm{Y}$-intercept $q_{2 \mid\{2\}}$

$$
\frac{x}{q_{1 \mid\{1\}}}+\frac{y}{q_{2 \mid\{2\}}}=1
$$

as shown in Fig. 17. By $(2) \Longleftrightarrow(3)$, if the stability region is polyhedral, then (18) holds. Also, the point of intersection of the lines is $\left(q_{1 \mid\{1\}}-Q_{1}, q_{2 \mid\{2\}}-Q_{2}\right)$. So, we have

$$
\begin{aligned}
\frac{\left(q_{1 \mid\{1\}}-Q_{1}\right)}{q_{1 \mid\{1\}}}+ & \frac{\left(q_{2 \mid\{2\}}-Q_{2}\right)}{q_{2 \mid\{2\}}} \\
& =2-\left(\frac{Q_{1}}{q_{1 \mid\{1\}}}+\frac{Q_{2}}{q_{2 \mid\{2\}}}\right) \geq 1
\end{aligned}
$$

which implies that the point $\left(q_{1 \mid\{1\}}-Q_{1}, q_{2 \mid\{2\}}-Q_{2}\right)$ lies above the line given by (62). Since the stability region is bounded by two lines, (63) is equivalent to the stability region being convex.

b) $\mathfrak{S}_{\mathrm{ALOHA}}$ is convex $\Longrightarrow \mathfrak{S}_{\mathrm{ALOHA}}$ is polyhedral: We will prove that the contrapositive is true. So, assume that the stability region is not a polyhedron. Then, by Proposition 1 (proved below) the stability region has a form where the boundary is a strictly convex function in the middle with or without lines at the ends. In any case, it follows that the stability region is not convex.

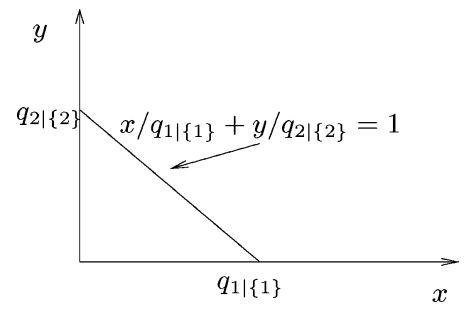

Fig. 17. The line with X-intercept $q_{1 \mid\{1\}}$ and $\mathrm{Y}$-intercept $q_{2 \mid\{2\}}$.

Proof That Part 3) Is Equivalent to Part 5):

We use Lemma 1. Substitute $\boldsymbol{p}=[1,1]$ in Lemma 1 . Then, $\lambda_{1}^{*}=q_{1 \mid\{1,2\}}$ and $\lambda_{2}^{*}=q_{2 \mid\{1,2\}}$ and it is easily seen that the stability region with $\boldsymbol{p}=[1,1]$ coincides with $\mathfrak{S}_{\text {ALOHA }}$ if and only if

$$
\frac{q_{1 \mid\{1,2\}}}{q_{1 \mid\{1\}}}+\frac{q_{2 \mid\{1,2\}}}{q_{2 \mid\{2\}}} \geq 1 .
$$

\section{Proof That Part 3) Is Equivalent to Part 4):}

It can be easily verifed that when (18) holds, $\mathfrak{S}_{\mathrm{ALOHA}}=\mathfrak{C}$. On the other hand, if (18) does not hold, then $\mathfrak{C}$ is a triangle with vertices $(0,0),\left(q_{1 \mid\{1\}}, 0\right)$, and $\left(0, q_{2 \mid\{2\}}\right)$ whereas from Lemma $2, \mathfrak{S}_{\mathrm{ALOHA}}$ lies strictly inside this triangle.

Next, we claim the following.

Proposition 1: If $\mathfrak{S}_{\mathrm{ALOHA}}$ is not polyhedral, then the strictly convex parts of $f$ which bound the regions $\mathcal{R}_{1}$ and $\mathcal{R}_{2}$ in Lemma 2 coincide on the boundary of the stability region.

Proof: Consider the equations

$$
\begin{aligned}
x=\left(\frac{q_{1 \mid\{1\}} q_{2 \mid\{2\}}-\sqrt{y Q_{1} q_{1 \mid\{1\}} q_{2 \mid\{2\}}}}{Q_{2}}\right) \\
\times\left(1-\sqrt{\frac{y Q_{1}}{q_{1 \mid\{1\}} q_{2 \mid\{2\}}}}\right)
\end{aligned}
$$

and

$$
\begin{aligned}
y=\left(\frac{q_{1 \mid\{1\}} q_{2 \mid\{2\}}-\sqrt{x Q_{2} q_{1 \mid\{1\}} q_{2 \mid\{2\}}}}{Q_{1}}\right) \\
\quad \times\left(1-\sqrt{\frac{x Q_{2}}{q_{1 \mid\{1\}} q_{2 \mid\{2\}}}}\right)
\end{aligned}
$$

which are the strictly convex parts of $f$ characterizing regions $\mathcal{R}_{2}$ and $\mathcal{R}_{1}$, respectively. If we make the substitution $x^{\prime}=$ $\sqrt{x Q_{2} q_{1 \mid\{1\}} q_{2 \mid\{2\}}}$ and $y^{\prime}=\sqrt{y Q_{1} q_{1 \mid\{1\}} q_{2 \mid\{2\}}}$, then (64) and (65) become

$\left(q_{1 \mid\{1\}} q_{2 \mid\{2\}}-y^{\prime}\right)^{2}=x^{\prime 2} \quad$ and $\quad\left(q_{1 \mid\{1\}} q_{2 \mid\{2\}}-x^{\prime}\right)^{2}=y^{\prime 2}$.

Solving the equations in (66) simultaneously gives

$$
x^{\prime}+y^{\prime}=q_{1 \mid\{1\}} q_{2 \mid\{2\}} \text {. }
$$

It follows that any $\left(x^{*}, y^{*}\right)$ which satisfies (67) satisfies (66) as well. In other words, all the points $(x, y)$ which satisfy (64) also satisfy (65) and vice versa and we are done. 
The above claim guarantees that the nonlinear portions of the function $f$ in Lemma 2 coincide for regions $\mathcal{R}_{1}$ and $\mathcal{R}_{2}$. This implies that the nonlinear boundary of $\mathfrak{S}_{\mathrm{ALOHA}}$ is a strictly convex function since $f$ is strictly convex.

\section{Proof of Theorem 4}

From [30], it can be shown (as a special case) that for a finite $(N \geq 2)$-user system, with symmetric arrival rate, a symmetric MPR reception model as given by (19), and transmission probability $p$, under the following stochastic dominance condition:

$$
C_{1} \geq \frac{C_{2}}{2} \geq \cdots \geq \frac{C_{i}}{i} \geq \cdots \geq \frac{C_{N}}{N}
$$

the maximum stable throughput of ALOHA is given by

$$
\rho_{\mathrm{ALOHA}}(p)=\sum_{i=1}^{N}\left(\begin{array}{c}
N \\
i
\end{array}\right) p^{i}(1-p)^{N-i} C_{i}
$$

Now, note that

$$
\sum_{i=1}^{N}\left(\begin{array}{c}
N \\
i
\end{array}\right) p^{i}(1-p)^{N-i} C_{i} \leq \max \left\{C_{1}, \ldots, C_{N}\right\}
$$

Clearly, if $C_{N}=\max \left\{C_{1}, \ldots, C_{N}\right\}$ then the above inequality is satisfied with equality for $p=1$. By Corollary 1 , it follows that $\rho_{\text {ALOHA }}(1)=\rho_{\text {ALOHA }}=\rho=\max \left\{C_{1}, \ldots, C_{N}\right\}$. The converse follows directly.

\section{E. Proof of Theorem 6}

Let $Q_{i}^{t}$ denote the queue length of the $i$ th user in time slot $t$. Let $\beta_{i}^{t} \stackrel{\text { i.i.d }}{\sim} \operatorname{Bernoulli}(r)$, denote the number of packets that arrive at the $i$ th users' queue in time slot $t$. Let $F(x, y)$ be the moment-generating function of the joint arrival process. Thus, $\forall|x| \leq 1,|y| \leq 1, t \in \mathbb{N}$

$$
F(x, y)=\mathbb{E}\left(x^{\beta_{1}^{t}} y^{\beta_{2}^{t}}\right)=(x r+\bar{r})(y r+\bar{r}) .
$$

Then, from the queue evolution (5), it can be seen that

$$
\begin{aligned}
\mathbb{E} & \left(x_{1}^{Q_{1}^{(t+1)}} y^{Q_{2}^{(t+1)}}\right) \\
= & F(x, y)\left[\mathbb{E}\left(\mathbf{1}\left[Q_{1}^{t}=0, Q_{2}^{t}=0\right]\right)\right. \\
& +\left(\frac{p a}{x}+p \bar{a}+\bar{p}\right) \mathbb{E}\left(x^{Q_{1}^{t}} \mathbf{1}\left[Q_{1}^{t}>0, Q_{2}^{t}=0\right]\right) \\
& +\left(\frac{p a}{y}+p \bar{a}+\bar{p}\right) \mathbb{E}\left(y^{Q_{2}^{t}} \mathbf{1}\left[Q_{1}^{t}=0, Q_{2}^{t}>0\right]\right) \\
& +\left(\left(p a+p^{2}(b-a)\right)\left(\frac{1}{x}+\frac{1}{y}\right)+\bar{p}^{2}+2 \bar{p} p \bar{a}+p^{2}(1-2 b)\right) \\
& \left.\times \mathbb{E}\left(x^{Q_{1}^{t}} y^{Q_{2}^{t}} \mathbf{1}\left[Q_{1}^{t}>0, Q_{2}^{t}>0\right]\right)\right] .
\end{aligned}
$$

By Lemma 1, if $r<\left(p a+p^{2}(b-a)\right)$, then the ALOHA system is stable. Since, $\left(Q_{1}^{t}, Q_{2}^{t}\right)$ is an irreducible, aperiodic Markov chain, stability is equivalent to existence of a unique stationary (limiting) distribution. Let $G(x, y)$ be the moment-generating function of the joint stationary queue process, viz.,

$$
G(x, y)=\lim _{t \rightarrow \infty} \mathbb{E}\left(x^{Q_{1}^{t}} y^{Q_{2}^{t}}\right)
$$

Now, note that

$$
\begin{aligned}
\lim _{t \rightarrow \infty} \mathbb{E}\left(\mathbf{1}\left[Q_{1}^{t}=0, Q_{2}^{t}=0\right]\right) & =G(0,0) \\
\lim _{t \rightarrow \infty} \mathbb{E}\left(x^{Q_{1}^{t}} \mathbf{1}\left[Q_{1}^{t}>0, Q_{2}^{t}=0\right]\right) & =G(x, 0)-G(0,0) \\
\lim _{t \rightarrow \infty} \mathbb{E}\left(y^{Q_{2}^{t}} \mathbf{1}\left[Q_{1}^{t}=0, Q_{2}^{t}>0\right]\right)= & G(0, y)-G(0,0) \\
\lim _{t \rightarrow \infty} \mathbb{E}\left(x^{Q_{1}^{t}} y^{Q_{2}^{t}} \mathbf{1}\left[Q_{1}^{t}>0, Q_{2}^{t}>0\right]\right)= & G(x, y)+G(0,0) \\
& -G(x, 0)-G(0, y) .
\end{aligned}
$$

Using an approach similar to ([23], [24]), from (72) it follows that $G(x, y)$ satisfies the following functional equation:

$$
\begin{aligned}
G(x, y)= & F(x, y)(A(x, y)(G(x, 0)-G(0,0)) \\
& +C(x, y)(G(x, y)+G(0,0)-G(x, 0)-G(0, y)) \\
& +B(x, y)(G(0, y)-G(0,0))+G(0,0))
\end{aligned}
$$

where

$$
\begin{aligned}
A(x, y)= & \frac{p a}{x}+p(1-a)+(1-p) \\
B(x, y)= & \frac{p a}{y}+p(1-a)+(1-p) \\
C(x, y)= & \left(p^{2} b+p \bar{p} a\right)\left(\frac{1}{x}+\frac{1}{y}\right) \\
& +\bar{p}^{2}+2 p \bar{p}(1-a)+p^{2}(1-2 b) .
\end{aligned}
$$

As observed by Sidi [23], the above functional equation cannot be solved for $G(x, y)$ easily. However, (74) can be used to compute the average delay as follows. First, use the fact that $G(1,1)=1$ and $G(1,0)=G(0,1)$ (symmetry) to find

$$
\begin{aligned}
G(1,0)\left(2 p^{2}(b-a)+p a\right)-G( & 0,0) p^{2}(b-a) \\
& =p a+p^{2}(b-a)-r .
\end{aligned}
$$

Let $G_{1}(x, y) \triangleq d G(x, y) / d x$. From (74), we can show that

$$
G_{1}(1,1)=\frac{p^{2}(b-a) G_{1}(1,0)+r \bar{r}}{p a+p^{2}(b-a)-r}
$$

and

$$
\begin{aligned}
\left.\frac{d G(x, x)}{d x}\right|_{x=1}=2 r & +\frac{\left(p a+2 p^{2}(b-a)\right) G_{1}(1,0)}{p a+p^{2}(b-a)-r} \\
& +\frac{\left(r^{2}+2 r-4 r\left(p^{2}(b-a)+p a\right)\right)}{2\left(p a+p^{2}(b-a)-r\right)} .
\end{aligned}
$$

It follows by symmetry that

$$
\left.\frac{d G(x, x)}{d x}\right|_{x=1}=2 G_{1}(1,1) .
$$


Substituting (79) in (78) and eliminating $G_{1}(1,0)$ from (77) and (78) gives

$$
G_{1}(1,1)=\frac{1}{p a}\left[r+\frac{\left(2 r^{2}-2 r^{2} p a-r^{2} p^{2}(b-a)\right)}{2\left(p a+p^{2}(b-a)-r\right)}\right] .
$$

Since $G_{1}(1,1)$ is equal to the mean queue length of the users, a simple application of Little's theorem gives us the mean queueing delay $(D)$ as

$$
\begin{aligned}
D & =\frac{G_{1}(1,1)}{r} \\
& =\frac{1}{p a}\left[1+\frac{\left(2 r-2 r p a-r p^{2}(b-a)\right)}{2\left(p a+p^{2}(b-a)-r\right)}\right] \\
& =\frac{1}{a}\left[\frac{a(1-r)+p(b-a)(1-r / 2)}{p a+p^{2}(b-a)-r}\right] .
\end{aligned}
$$

\section{ACKNOWLEDGMENT}

V. Naware would like to thank S. Mahajan for some useful discussions on nonlinear optimization problems.

\section{REFERENCES}

[1] N. Abramson, "The ALOHA system-Another alternative for computer communications," in Proc. Fall Joint Computer Conf., AFIPS Conf. Proc., vol. 44, Montvale, NJ., 1970, pp. 281-285.

[2] B. Tsybakov and W. Mikhailov, "Ergodicity of slotted ALOHA systems," Probl. Inform. Transm., vol. 15, no. 4, pp. 73-87, 1979.

[3] R. Rao and A. Ephremides, "On the stability of interacting queues in a multi-access system," IEEE Trans. Inf. Theory, vol. 34, no. 5, pp. 918-930, Sep. 1988.

[4] W. Szpankowski, "Stability conditions for some multiqueue distributed systems: Buffered random access systems," Adv. Appl. Probab., vol. 26, pp. 498-515, 1994.

[5] W. Luo and A. Ephremides, "Stability of $N$ interacting queues in random-access systems," IEEE Trans. Inf. Theory, vol. 45, no. 5, pp. 1579-1587, Jul. 1999.

[6] V. Anantharam, "Stability region of the finite-user slotted ALOHA protocol," IEEE Trans. Inf. Theory, vol. 37, no. 3, pp. 535-540, May 1991.

[7] S. Ghez, S. Verdú, and S. Schwartz, "Stability properties of slotted ALOHA with multipacket reception capability," IEEE Trans. Autom. Control, vol. 33, no. 7, pp. 640-649, Jul. 1988.

[8] - "Optimal decentralized control in the random access multipacket channel," IEEE Trans. Autom. Control, vol. 34, no. 11, pp. 1153-1163, Nov. 1989.

[9] J. Sant and V. Sharma, "Performance analysis of a slotted-ALOHA protocol on a capture channel with fading," Queueing Systems, Theory Applic., vol. 34, no. 1, pp. 1-35, 2000.

[10] S. Adireddy and L. Tong, "Exploiting decentralized channel state information for random access," IEEE Trans. Inf. Theory, vol. 51, no. 2, pp. 537-561, Feb. 2005.
[11] G. Mergen and L. Tong, "Stability and capacity of regular wireless networks," IEEE Trans. Inf. Theory, vol. 51, no. 6, pp. 1938-1953, Jun. 2005.

[12] Q. Zhao and L. Tong, "A multiqueue service room MAC protocol for wireless networks with multipacket reception," IEEE/ACM Trans. Netw., vol. 11, no. 1, pp. 125-137, Feb. 2003.

[13] _ - "A dynamic queue protocol for multiaccess wireless networks with multipacket reception," IEEE Trans. Wireless Commun., vol. 3, no. 6, pp. 2221-2231, Nov. 2004.

[14] V. Naware, G. Mergen, and L. Tong, "Stability and Delay of Finite User Slotted ALOHA with Multipacket Reception," Cornell Univ., Ithaca, NY, Tech. Rep. ACSP-TR-08-04-01, 2004.

[15] M. Grossglauser and D. N. C. Tse, "Mobility increases the capacity of wireless adhoc networks," IEEE/ACM Trans. Netw., vol. 10, no. 4, pp. 477-486, Aug. 2002.

[16] S. Resnick, Adventures in Stochastic Processes. Boston, MA: Birkhäuser, 1994.

[17] R. M. Loynes, "The stability of a queue with nonindependant inter-arrival and service times," in Proc. Cambridge Philos. Soc., vol. 58, 1962, pp. 497-520.

[18] G. Mergen and L. Tong, "Stability and Capacity of Wireless Networks with Probabilistic Peceptions: Part I-General Topologies, Part II-Regular Networks," Cornell Univ., Ithaca, NY, Tech. Rep. ACSP-TR-01-03-01, 2003.

[19] P. Viswanath, D. N. C. Tse, and R. Laroia, "Opportunistic beamforming using dumb antennas," IEEE Trans. Inf. Theory, vol. 48, no. 1, pp. 1277-94, Jun. 2002.

[20] A. Ephremides and B. Hajek, "Information theory and communication networks: An unconsummated union," IEEE Trans. Inf. Theory, vol. 44, no. 6, pp. 2416-2434, Oct. 1998.

[21] W. Szpankowski, "Bounds for queue lengths in a contention packet broadcast system," IEEE Trans. Commun., vol. COM-34, no. 11, pp. 1132-1140, Nov. 1986.

[22] V. Naware and L. Tong, "Using queue statistics for beamforming in ALOHA," in Proc. Asilomar Conf. Signals, Systems and Computers, Monterey, CA, Nov. 2003, pp. 212-215.

[23] M. Sidi and A. Segall, "Two interfering queues in packet-radio networks," IEEE Trans. Commun., vol. COM-31, no. 1, pp. 123-129, Jan. 1983.

[24] P. Nain, "Analysis of a two node ALOHA network with infinite capacity buffers," in Proc. Int. Sem. Computer Networking, Performance Evaluation, Tokyo, Japan, 1985, pp. 2.2.1-2.2.16.

[25] G. Fayolle and R. Iasnogorodski, "Two coupled processors: The reduction to a Riemann-Hilbert problem," Z. Wahrscheinlichkeitstheorie, vol. 47, pp. 325-351, 1979.

[26] J. W. Cohen and O. J. Boxma, Boundary Value Problems in Queueing System Analysis. Amsterdam, The Netherlands: North-Holland, 1983.

[27] H. Takagi and L. Kleinrock, "Mean packet queueing delay in a buffered two-user CSMA/CD system," IEEE Trans. Commun., vol. COM-33, pp. 1136-1139, Oct. 1985.

[28] T. N. Saadawi and A. Ephremides, "Analysis, stability and optimization of slotted ALOHA with finite number of buffered users," IEEE Trans. Autom. Control, vol. AC-26, no. 3, pp. 680-689, Jun. 1981.

[29] A. Ephremides and R.-Z. Zhu, "Delay analysis of interacting queues with an approximate model," IEEE Trans. Commun., vol. COM-35, no. 2, pp. 194-201, Feb. 1987.

[30] S. Adireddy and L. Tong, "Optimal transmission probabilities for slotted ALOHA in fading channels," in Proc. Conference on Information Sciences and Systems (CISS'02), Princeton, NJ, Mar. 2002. 